Inhomogeneity-induced cosmic acceleration in a dust universe

This article has been downloaded from IOPscience. Please scroll down to see the full text article.

2008 Class. Quantum Grav. 25175001

(http://iopscience.iop.org/0264-9381/25/17/175001)

The Table of Contents and more related content is available

Download details:

IP Address: 140.112.113.225

The article was downloaded on 17/07/2009 at 08:47

Please note that terms and conditions apply. 


\title{
Inhomogeneity-induced cosmic acceleration in a dust universe
}

\author{
Chia-Hsun Chuang ${ }^{1}$, Je-An Gu ${ }^{2}$ and W-Y P Hwang \\ Department of Physics, National Taiwan University, Taipei 10617, Taiwan, Republic of China \\ E-mail: r92222016@ntu.edu.tw,jagu@phys.cts.nthu.edu.tw and wyhwang@phys.ntu.edu.tw
}

Received 22 February 2008, in final form 4 June 2008

Published 13 August 2008

Online at stacks.iop.org/CQG/25/175001

\begin{abstract}
It is the common consensus that the expansion of a universe always slows down if the gravity provided by the energy sources therein is attractive and accordingly one needs to invoke dark energy as a source of anti-gravity for understanding the cosmic acceleration. To examine this point we find counterexamples for a spherically symmetric dust fluid described by the Lemaittre-Tolman-Bondi solution without singularity. Thus, the validity of this naive consensus is indeed doubtful and the effects of inhomogeneities should be restudied. These counter-intuitive examples open a new perspective on the understanding of the evolution of our universe.
\end{abstract}

PACS numbers: 98.80.Jk, 98.80.Es, 95.36.+x, 04.20.Jb

\section{Introduction}

In 1998, via the distance measurements of type Ia supernovae, it was discovered that the expansion of the universe is accelerating $[1,2]$. The accelerating expansion of the present universe was reinforced recently by the updated supernova data [3-7] and the WMAP measurement [8] of cosmic microwave background (CMB). Regarding the cosmic evolution, it is the common consensus that normal matter (such as protons, neutrons, electrons, etc) can only provide attractive gravity and therefore should always slow down the cosmic expansion, i.e.,

$$
\text { Normal matter } \Rightarrow \text { Attractive gravity } \Rightarrow \text { Deceleration. }
$$

Thus, to explain this surprising, mysterious phenomenon of the accelerating expansion, many people rely on exotic energy sources, as generally called 'dark energy', which provide significant negative pressure and accordingly anti-gravity (repulsive gravity).

1 Present address: Homer L Dodge Department of Physics and Astronomy, University of Oklahoma, 440 W Brooks St., Norman, OK 73019, USA.

2 Present address: Physics Division, National Center for Theoretical Sciences, PO Box 2-131, Hsinchu, Taiwan. 
The above conclusion about the existence of the cosmic acceleration and the necessity of introducing dark energy is based on a simplified cosmological model, the FriedmannLemaitre-Robertson-Walker (FLRW) model, and indeed could be model dependent. In the FLRW model the universe is assumed to be homogeneous and isotropic (i.e. the cosmological principle) and accordingly the Robertson-Walker (RW) metric is invoked in the Einstein equations that describe the evolution of the universe. Meanwhile the energy-momentum tensor in the right-hand side of the Einstein equations is regarded to truly reflect the real energy distribution (averaged in space) of the universe. Nevertheless, so far there is no convincing proof validating this simplification.

Apparently, our universe at present is not homogeneous at small scales. The cosmological principle is roughly realized only at very large scales. To take advantage of the cosmological principle and invoke the RW metric, it is necessary to perform spatial averaging over large scales, along with which the form of the Einstein equations in general should change because of the nonlinearity of the Einstein equations [9]. That is, (i) when invoking the RW metric and the real energy sources of our universe in the Einstein equations for describing the cosmic evolution, the left-hand side (the geometry part) of the Einstein equations should be modified, or, from another point of view, (ii) if one insists on using the Einstein tensor corresponding to the RW metric in the left-hand side of the Einstein equations (or, equivalently, moving the above-mentioned modification in the geometry part to the right-hand matter-energy part), there should appear new, effective energy sources coming from geometry (which is certainly 'dark') and consequently the energy-momentum tensor in the right-hand side does not truly correspond to the real energy distribution of our universe. Thus, generally speaking, it is doubtful to employ the Einstein equations to describe long-time, large-scale phenomena, such as the evolution of our universe, while the spatially averaged energy-momentum tensor and the spatially averaged metric tensor are used therein. (For more discussions about the validity and the problems of the FLRW cosmology, see [10].)

Instead of invoking exotic energy sources or unconfirmed physics, it is suggested [11-22] that the cosmic acceleration might originate from the violation of the cosmological principle, homogeneity and isotropy, i.e., the acceleration might be induced by the inhomogeneities of the universe. The possible change of the deceleration parameter in an inhomogeneous universe has been pointed out in [23, 24]. In particular, it has been shown that the luminosity distanceredshift relation indicated by the supernova data can be reproduced in an inhomogeneous cosmological model without introducing dark energy [25-34].

The current situation of our knowledge (what we know and what we do not know well) about observations, cosmic acceleration and inhomogeneities is as follows:

\section{Known:}

- Based on the FLRW cosmology, the current observational results indicate the existence of the cosmic acceleration. (That is, by invoking the FLRW cosmological model to interpret the observational data, one would conclude that the expansion of the universe is accelerating in the recent epoch.)

Doubts:

(1) Is the FLRW cosmology a good approximation?

(2) Do the current observational results indicate the existence of cosmic acceleration for the real universe with complicated energy distribution? (Even the definition of accelerating expansion is an issue for our complicated universe. This issue will be discussed in section 2.)

(3) Can the inhomogeneities of our universe explain the observational results?

(4) Can the inhomogeneities of our universe generate accelerating expansion? 
These four doubts are far from being fully answered. This is due to the difficulties from the complexity of the real energy distribution and the nonlinearity of the Einstein equations. Because of these difficulties, instead of dealing with the full Einstein equations describing the real complicated universe, usually people employ the following two approaches to sketch the possible answers to the above doubts:

(I) Taking a perturbative approach with a simple background (such as the homogeneous and isotropic RW background).

(II) Invoking exact solutions of the Einstein equations describing an inhomogeneous universe.

In addition to these two approaches, there are also non-perturbative studies without invoking exact solutions, e.g., see [35-37].

Via approach (I) with a homogenous and isotropic background, the positive answer to doubt (1) is supported in [38, 39], and the negative answer to doubts (3) and (4) supported in [38-41]. Nevertheless, the reliability of the perturbative approach in (I) is doubtful for investigating the late-time cosmic evolution, for which not only energy distribution, but also curvature, may not be described with perturbations [21, 42]. Furthermore, the arguments of [38-41] have been countered in [21, 43, 42, 37].

The drawback of approach (II) is that the exact solution invoked may be very different from the real situation of our universe. From one angle, instead of doubts (3) and (4), approach (II) is the answer to a more general question:

- Can inhomogeneities explain observational results and generate accelerating expansion? Or, more precisely,

$\left(3^{\prime}\right)$ Does there exist a universe (maybe very different from ours) in which the inhomogeneities can explain the observational results?

(4') Does there exist a universe in which the inhomogeneities can drive the expansion to accelerate?

Via approach (II), for doubt (3') it has been shown in [25-34] that the supernova data can be explained by invoking the Lemaitre-Tolman-Bondi solution [44-46] that describes a spherically symmetric (but inhomogeneous) dust fluid.

In the present work, we take approach (II) for investigating the general possibility of generating accelerating expansion via inhomogeneities, i.e. doubt (4'). Against the common intuition in equation (1) we find examples of accelerating expansion in the case of a spherically symmetric dust fluid described by the Lemaître-Tolman-Bondi (LTB) solution, thereby giving support to the positive answer to doubt $\left(4^{\prime}\right)$. In addition to our examples, Kai et al [47] also found acceleration examples based on the LTB solution, where there exists singularity around the center of the spherically symmetric system during the accelerating epoch. In contrast, in our examples the system is smooth everywhere and no singularity is involved. (For greater comparison, see section 5.) The possibility of the accelerating expansion in the LTB model was also pointed out by Paranjape and Singh in [48], in which numerical models exhibiting acceleration were constructed by an approximation where the contribution of 3-curvature dominates over the matter density.

There also existed acceleration examples for a system consisting of two or more regions [37, 42, 49-52]. In these acceleration examples, connecting the separate regions smoothly and the effect of the junction between these regions are the essential issues yet to be seriously explored. We particularly note that it requires much caution to connect two (or more) regions. In many cases the effect from the junction between separate regions is significant and should not be ignored. A similar doubt was also raised by Paranjape and Singh in [52]. As a demonstration of how things may go wrong when the connection or the junction is not appropriately taken 
care of, in appendix $\mathrm{C}$ we investigate in detail the acceleration examples studied by Nambu and Tanimoto in [49], and show that actually there should be no acceleration in those cases when we properly connect the separate regions and seriously take the effect of the junction into account.

The acceleration examples we find could be far away from the real situation of our universe. We are not proposing to employ these mathematical examples or the LTB solution to describe our universe. These acceleration examples, which provide a positive answer to doubt $\left(4^{\prime}\right)$, are to demonstrate how inhomogeneities can drive the expansion to accelerate, thereby showing how our intuition about the interplay of gravity and the cosmic evolution may go wrong (i.e. against the common intuition in equation (1)). Accordingly, the effects of inhomogeneities on the evolution of the universe should be carefully restudied.

This paper is organized as follows. In section 2, a tricky issue of the definition of acceleration is discussed and two definitions to be utilized for searching acceleration examples are introduced. In section 3, the LTB solution is described. In sections 4 and 5, we present the examples of the accelerating expansion corresponding to these two definitions of acceleration, respectively. A summary and discussions follow in section 6.

Throughout the present paper we will use the units where $c=8 \pi G=1$. We note that in this unit system there is still one unit unspecified, and, as a result, the value of one of the dimensionful quantities can be arbitrarily set. For example, in the acceleration examples we will present, the physical size of the spherical region under consideration can be any length long (such as 1 fermi, $1 \mathrm{~cm}, 1 \mathrm{pc}, 1 \mathrm{Mpc}$, Hubble length $H_{0}^{-1}$, etc). Once the value of one of the dimensionful quantities (which are independent of $c$ and $G$ ) is settled, all the units for the dimensionful quantities studied in the present work are specified.

\section{Definitions of acceleration}

In cosmology, expansion and acceleration of the universe are a subject associated with the evolution of the space (in size), and should have nothing to do with the particle motion relative to the space. How to define the speed and the acceleration purely corresponding to the evolution of the space, meanwhile avoiding confusion and interference from the particle motion relative to the space? This is a tricky issue for a real universe with complicated energy distribution [10].

To characterize the evolution status of the space, usually one needs to invoke two quantities: a length (distance) quantity $L$ and a time quantity $t$, with which $\dot{L}$ and $\ddot{L}$ (where the over-dot denotes the time derivative) present the speed and the acceleration of the expansion, respectively. The tricky issue mentioned above then corresponds to the problem of making the choice of these two quantities. With different choices one has different definitions. It will be puzzling if the description of the space evolution (expansion and acceleration) status is not universal but depends on the definition one invokes.

If it is inevitable to make the choice which the description of the evolution status of the universe is based on and maybe sensitively depends on, what is the reasonable physical choice? The very guiding principle for making the choice is that we are quantifying simply the evolution of the space and we need to make sure that the definition we invoke does not involve particle motion relative to the space nor something fake stemming from an inappropriate frame choice.

In this section we will introduce two definitions, as to be called line acceleration and domain acceleration, respectively, involving two length quantities: (i) the distance between two points in space and (ii) the size of a domain in space. For these two kinds of lengths the above tricky issue relates to the choice of two points for the line acceleration and the 
choice of the domain (i.e. its boundary) for the domain acceleration. With different choices (corresponding to different definitions) one might obtain very different conclusions about the acceleration/deceleration status.

In general, with a certain choice of frame (coordinate system), the two spatial points and the boundary mentioned above can be fixed in the spatial coordinate space. With an improper choice of these two points and the boundary, or, equivalently, with an improper choice of frame (in which the spatial coordinates of the two points and the boundary are fixed), the change of the length quantity with time may be incapable of representing the true evolution of the space, which could be mixed up with the evolution of the frame. The contribution from the frame evolution to the acceleration of the chosen length will be called 'frame acceleration' in the present paper.

To construct suitable definitions of acceleration for these two kinds of lengths, for simplicity we consider a universe consisting of freely-moving particles (i.e., moving along geodesics), among which there is no interaction other than gravity. In this case, for the line acceleration a simple reasonable length quantity to choose is the distance between two (freelymoving) particles. In contrast, an apparently improper definition which could lead to fake frame acceleration is to invoke the distance between two points which move 'outward' relative to the particles in between, i.e., accordingly, there are more and more particles between these two chosen spatial points.

As for the domain acceleration, a reasonable choice is the size of a spatial domain in which the number of particles is constant in time. In contrast, an apparently improper definition which could lead to fake frame acceleration is to invoke a domain whose boundary moves outward relative to the particles therein, i.e., accordingly, there are more and more particles within this domain.

For implementing these two acceleration definitions, meanwhile satisfying the abovementioned requirements for avoiding the confusion from particle motion and fake frame acceleration, it is particularly beneficial to use the synchronous gauge. Note that in a dust universe the synchronous gauge can be chosen if and only if the vorticity vanishes. In the synchronous gauge the line element is as follows:

$$
\mathrm{d} s^{2}=-\mathrm{d} t^{2}+h_{i j}(\mathbf{x}, t) \mathrm{d} x^{i} \mathrm{~d} x^{j},
$$

where $t$ is the cosmic time. In this gauge, the cosmic time $t$ is simple and universal to choose in defining acceleration. Regarding the length quantity, in this gauge the above-mentioned requirements are easy to meet because a point fixed in the spatial coordinate space is a geodesic. In particular, for a dust fluid with the energy-momentum tensor

$$
T^{\mu \nu}=\rho u^{\mu} u^{\nu}, \quad u^{\mu}=(1,0,0,0),
$$

the distance between two fixed points and the size of a domain with its boundary fixed in this coordinate space are simple and direct choices satisfying the requirements. In the following, we will focus on this simple case of a dust fluid described in the synchronous gauge, and introduce two definitions of acceleration involving these two length quantities, respectively.

\subsection{Line acceleration}

Regarding two points in space with the proper distance $L(t)$ between them at time $t$, it is reasonable to use $\dot{L}(t)$ and $\ddot{L}(t)$ to characterize the expansion/collapse status and the acceleration/deceleration status of the space in between. The expansion rate and the deceleration parameter for the proper distance $L(t)$ are defined in the usual way as follows:

$$
H_{L} \equiv \dot{L} / L
$$




$$
q_{L} \equiv-\frac{\ddot{L} / L}{H_{L}^{2}}=-\frac{\ddot{L} L}{\dot{L}^{2}} .
$$

The condition $\left\{H_{L}>0, q_{L}<0\right\}$ corresponds to the accelerating expansion of the proper distance between these two points in space, which is dubbed 'line acceleration' in the present paper. We have found examples of the line acceleration in a dust universe, as will be shown in section 4 .

\subsection{Domain acceleration}

Another definition of acceleration, as dubbed 'domain acceleration' in the present paper, has been widely used in the literature $[11,21,35-37,42,49-54]$. It is for a spatial domain $D$ with a finite volume

$$
V_{D} \equiv \int_{D} \sqrt{h} \mathrm{~d}^{3} x
$$

where $h$ is the determinant of the spatial metric tensor $h_{i j}$. Invoking the length scale of the domain,

$$
L_{D} \equiv V_{D}^{1 / 3},
$$

one can define the expansion rate and the deceleration parameter of the domain in the usual way as follows:

$$
\begin{aligned}
& H_{D} \equiv \dot{L}_{D} / L_{D}, \\
& q_{D} \equiv-\frac{\ddot{L}_{D} / L_{D}}{H_{D}^{2}}=-\frac{\ddot{L}_{D} L_{D}}{\dot{L}_{D}^{2}} .
\end{aligned}
$$

The condition $\left\{H_{D}>0, q_{D}<0\right\}$ corresponds to the accelerating expansion of the domain (in size), i.e., domain acceleration.

As shown in [55-58], for an infinitesimal domain in a dust universe without vorticity the deceleration parameter $q_{D}$ is always positive, i.e., corresponding to local deceleration. Nevertheless, the non-local deceleration/acceleration status of a domain with a nonzero finite volume (in particular, the observational universe of the Hubble size) may be very different [21]. So far there is no no-go theorem excluding the possibility of negative $q_{D}$. In contrast, we have found examples of the domain acceleration in a dust universe, as to be shown in section 5 .

We note that, in the special case of a homogeneous and isotropic universe described by the RW metric with the scale factor $a(t)$, the expansion rates and the deceleration parameters defined above are the same as those in the standard cosmology,

$$
\begin{aligned}
& H \equiv \dot{a} / a \\
& q \equiv-\frac{\ddot{a} / a}{H^{2}}=-\frac{\ddot{a} a}{\dot{a}^{2}}
\end{aligned}
$$

In the following section we will introduce the LTB solution, based on which we find examples of acceleration. (Reminder: the units where $c=8 \pi G=1$ will be employed.) 


\section{Lemaître-Tolman-Bondi (LTB) solution}

The LTB solution [44-46] is an exact solution of the Einstein equations for a spherically symmetric dust fluid. The metric is given by

$$
\mathrm{d} s^{2}=-\mathrm{d} t^{2}+\frac{\left(R,{ }_{r}\right)^{2} \mathrm{~d} r^{2}}{1+2 E(r)}+R^{2} \mathrm{~d} \Omega^{2},
$$

where $R$ is a function of the time coordinate $t$ and the radial coordinate $r, E(r)$ is an arbitrary function of $r$, and $R,{ }_{r}$ denotes the partial derivative of $R$ with respect to $r$. With this metric the Einstein equations can be reduced to two equations,

$$
\begin{aligned}
& \left(\frac{\dot{R}}{R}\right)^{2}=\frac{2 E(r)}{R^{2}}+\frac{2 M(r)}{R^{3}}, \\
& \rho(t, r)=\frac{2 M^{\prime}(r)}{R^{2} R, r}
\end{aligned}
$$

where $M(r)$ is an arbitrary function of $r$ and the over-dot denotes the partial derivative with respect to $t$. The solution of equation (13) can be written parametrically by using a variable $\eta=\int \mathrm{d} t / R$ as follows:

$$
\begin{aligned}
& R(\eta, r)=\frac{M(r)}{-2 E(r)}[1-\cos (\sqrt{-2 E(r)} \eta)] \\
& t(\eta, r)=\frac{M(r)}{-2 E(r)}\left[\eta-\frac{1}{\sqrt{-2 E(r)}} \sin (\sqrt{-2 E(r)} \eta)\right]+t_{b}(r),
\end{aligned}
$$

where $t_{b}(r)$ is an arbitrary function of $r$. Summarily, there is one dynamical field, $R(t, r)$, and three arbitrary functions, $E(r), M(r)$ and $t_{b}(r)$. For a given set of the three functions $\left\{E(r), M(r), t_{b}(r)\right\}$, we have a solution $R(t, r)$ specified by equations (15) and (16).

Regarding the behavior of the dynamical field and the three functions introduced above, it is reasonable to consider the requirements that there is no hole and no singularity in space and the energy density is non-negative and finite.

(1) For no hole at the center (i.e. the area of the spherical surface at $r=r_{0}$ goes to zero when $r_{0}$ goes to zero), $R(t, r=0)=0$.

(2) For no singularity, we consider $R, r(t, r) \neq 0$.

(3) For a non-negative and finite energy density, $0 \leqslant \rho(t, r)<\infty$.

According to these requirements, the dynamical field and the functions in the LTB solution should satisfy the following restrictions:

$$
\begin{array}{ll}
r=0: & R, \ddot{R}, M, E=0, \\
r>0: & R R, r>0, \quad M M^{\prime} \geqslant 0 .
\end{array}
$$

Without losing generality, in our study and in the remaining of the present paper we choose

$$
R, R, r, M, M^{\prime} \geqslant 0 .
$$

From the relation

$$
\ddot{R}=-\frac{M(r)}{R^{2}},
$$


as derived from the Einstein equation (13), the sign choice in equation (19) implies

$$
\ddot{R} \leqslant 0 .
$$

For more details about the restrictions on the LTB solution and the corresponding features of the dynamical field and the functions presented above, see appendix A. Note that the necessary and sufficient conditions of no shell crossing in a period of time are presented by Hellaby and Lake in [59]. In the present paper we consider the absence of shell crossing at some time, and accordingly the above-mentioned conditions are necessary conditions.

By introducing the following variables:

$$
a(t, r)=\frac{R(t, r)}{r}, \quad k(r)=-\frac{2 E(r)}{r^{2}}, \quad \rho_{0}(r)=\frac{6 M(r)}{r^{3}},
$$

the line element in equation (12) and the Einstein equations (13) and (14) can be rewritten in a form more similar to that of the RW metric,

$$
\begin{aligned}
& \mathrm{d} s^{2}=-\mathrm{d} t^{2}+a^{2}\left[\left(1+\frac{a, r}{a}\right)^{2} \frac{\mathrm{d} r^{2}}{1-k(r) r^{2}}+r^{2} \mathrm{~d} \Omega_{2}^{2}\right] \\
& \left(\frac{\dot{a}}{a}\right)^{2}=-\frac{k(r)}{a^{2}}+\frac{\rho_{0}(r)}{3 a^{3}}, \\
& \rho(t, r)=\frac{\left(\rho_{0} r^{3}\right)^{\prime}}{3 a^{2} r^{2}(a r)_{, r}} .
\end{aligned}
$$

The solution in equations (15) and (16) becomes

$$
\begin{aligned}
& a(\tilde{\eta}, r)=\frac{\rho_{0}(r)}{6 k(r)}[1-\cos (\sqrt{k(r)} \tilde{\eta})] \\
& t(\tilde{\eta}, r)=\frac{\rho_{0}(r)}{6 k(r)}\left[\tilde{\eta}-\frac{1}{\sqrt{k(r)}} \sin (\sqrt{k(r)} \tilde{\eta})\right]+t_{b}(r),
\end{aligned}
$$

where $\tilde{\eta} \equiv \eta r=\int \mathrm{d} t / a$.

Now the dynamical field describing the evolution of the space is $a(t, r)$ and the three functions to be given for specifying a solution $a(t, r)$ are $k(r), \rho_{0}(r)$ and $t_{b}(r)$ (corresponding to $E(r), M(r)$ and $t_{b}(r)$, respectively). Apparently, when all the functions $a(t, r), k(r), \rho_{0}(r)$ and $t_{b}(r)$ have no dependence on the radial coordinate $r$, we come back to the RW metric from equation (23), the Friedmann equation from equation (24) and the formula realizing stressenergy conservation from equation (25). From the comparison with the RW metric, we can get a rough picture of the LTB metric: $a(t, r)$ plays the role of a spatially varying, time-dependent scale factor describing the evolution of the space, $k(r)$ corresponds to the spatial curvature, $\rho_{0}(r)$ relates to the physical energy density $\rho(t, r)$, and $t_{b}\left(r_{1}\right)$ can be regarded as the initial time of the big bang at $r=r_{1}$, i.e., the space at $r=r_{1}$ starts to expand from singularity $\left[a\left(t_{b}, r_{1}\right)=0\right]$ at the time $t=t_{b}\left(r_{1}\right)$.

In search of examples of accelerating expansion in a dust universe described by the LTB solution, we tried a variety of LTB solutions corresponding to different choices of the functions $\left\{k(r), \rho_{0}(r), t_{b}(r)\right\}$, and eventually found examples among these tedious trials, as to be studied in the following two sections. We note that there is redundancy in the choices of the functions $\left\{E(r), M(r), t_{b}(r)\right\}$ or $\left\{k(r), \rho_{0}(r), t_{b}(r)\right\}$. For example, for a monotonically increasing function $M(r) \equiv \rho_{0}(r) r^{3}$, one can choose, without losing generality,

$$
\rho_{0}(r)=\text { constant, }
$$

which is the choice invoked in our search for the acceleration examples. 


\section{Line acceleration induced by inhomogeneities}

Regarding the demonstration of how the acceleration can be induced by inhomogeneity, naively we have a better chance with larger inhomogeneity which may induce more significant acceleration. In the LTB solution, due to the spherical symmetry, the inhomogeneity lies along the radial direction, while there is no inhomogeneity and also no acceleration (as implied by equation (21)) in the angular directions. Accordingly the possible line acceleration induced by inhomogeneity must involve the length component in the radial direction. For simplicity, we focus on the proper distance between the origin $(r=0)$ and the point at $r=r_{L}$ at time $t$,

$$
L_{r}(t) \equiv \int_{0}^{r_{L}} \sqrt{g_{r r}} \mathrm{~d} r
$$

where

$$
g_{r r}=\frac{(R, r)^{2}}{1+2 E(r)}=\frac{\left(a+a,{ }_{r} r\right)^{2}}{1-k(r) r^{2}} .
$$

The deceleration parameter corresponding to this radial proper distance is

$$
q_{r} \equiv-\frac{\ddot{L}_{r} L_{r}}{\dot{L}_{r}^{2}} .
$$

The sign of the deceleration parameter $q_{r}$ is determined by the sign of $\ddot{L}_{r}$ or, more precisely, the integral of $\partial_{t}^{2} \sqrt{g_{r r}}$ from the origin $r=0$ to $r=r_{L}$, i.e., $\int_{0}^{r_{L}} \partial_{t}^{2} \sqrt{g_{r r}} \mathrm{~d} r$.

In the LTB solution, inhomogeneity can be introduced by choosing inhomogeneous functions for $k(r), \rho_{0}(r)$ and $t_{b}(r)$. In this section, for simplicity we introduce inhomogeneity through only $k(r)$ by employing the following function:

$$
k(r)=-\frac{\left(h_{k}+1\right)\left(r / r_{k}\right)^{n_{k}}}{1+\left(r / r_{k}\right)^{n_{k}}}+1,
$$

while choosing

$$
\begin{aligned}
& \rho_{0}(r)=\text { constant } \\
& t_{b}(r)=0
\end{aligned}
$$

The behavior of the function $k(r)$ in equation (32) is illustrated in figure 1. For a large power $n_{k}$, this $k(r)$ function mimics a step function with violent change around $r=r_{k}$, accompanying which large inhomogeneity is introduced. We note that when $k(r)=0=E(r)$ (with arbitrary $\rho_{0}(r)$ and $\left.t_{b}(r)\right)$ there is no line acceleration (see appendix B), and therefore the inhomogeneity in $k(r)$ seems to play an essential role in generating accelerating expansion.

With the above choice of the three arbitrary functions in the LTB solution, we have six free parameters to tune,

$$
\left(t, r_{L}, \rho_{0}, r_{k}, n_{k}, h_{k}\right)
$$

In search of examples of the line acceleration in the radial direction, we surveyed this sixdimensional parameter space and did find examples eventually ${ }^{3}$. Table 1 presents one of the examples with significant acceleration, $-q_{r} \cong 0.834 \sim \mathcal{O}(1)$. We note that, as suggested by the observational data with the analyses based on the FLRW cosmology, the deceleration parameter of our present universe is of the order unity and is negative in sign.

${ }^{3}$ For $1-k(r) r^{2}$ to be positive, we consider two different sufficient conditions, $\left\{n_{k}>0, h_{k}>1, r_{k}<1\right\}$ and $\left\{h_{k}>-1, r \leqslant r_{L} \leqslant 1\right\}$, and restrict our search in the cases satisfying one of them. 


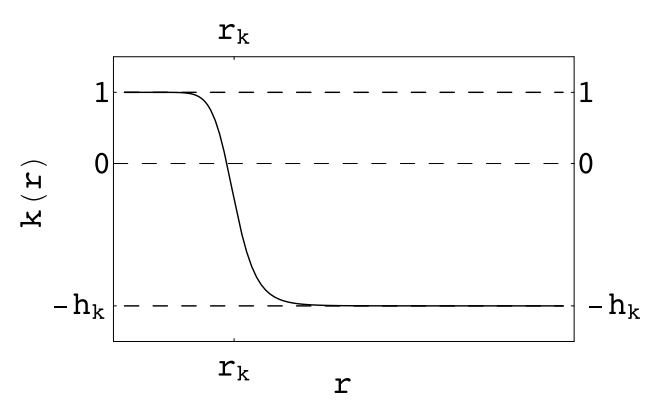

Figure 1. The plot of the function $k(r)$ invoked in the search for acceleration examples.

Table 1. One example of the line acceleration in the radial direction.

\begin{tabular}{llllllllll}
\hline$t$ & $r_{L}$ & $\rho_{0}$ & $r_{k}$ & $n_{k}$ & $h_{k}$ & $L_{r}$ & $\dot{L}_{r}$ & $\ddot{L}_{r}$ & $q_{r}$ \\
\hline 1 & 1 & 1 & 0.7 & 20 & 1 & 1.29 & 1.16 & 0.868 & -0.834 \\
\hline
\end{tabular}

The further details of the example in table 1 are illustrated in figure 2, which presents the energy density distribution and the acceleration/deceleration status of the radial and the angular line elements. As shown in this figure, the spherically symmetric dust fluid consists of three regions: two roughly homogeneous regions- the inner over-density region with positive $k(r)$ and smaller $a(t, r)$ and the outer under-density region with negative $k(r)$ and larger $a(t, r)$-and one transition or junction region, where the inhomogeneity locates, of those two homogeneous regions ${ }^{4}$. In the inhomogeneous region with significantly changing energy density we have acceleration in the radial line elements, i.e., $\partial_{t}^{2} \sqrt{g_{r r}}>0$, while in the homogeneous regions with smoothly distributed energy density we have deceleration. This result strongly supports the suggestion that inhomogeneity can induce accelerating expansion. In addition, as expected there is always no acceleration in the angular directions along which everything is uniformly distributed ${ }^{5}$.

For a demonstration of the scales of the size and other quantities of this system, in table 2 we fix the only one unspecified unit by using the length unit: $0.1 \mathrm{Mpc}, 1 \mathrm{Mpc}$, $10 \mathrm{Mpc}$ and $100 \mathrm{Mpc}$, and present the values of several dimensionful quantities, respectively. Among these quantities, $t$ corresponds to the time under consideration, $L_{r}$ the size of the system, $\rho(r=0)$ roughly the energy density of the inner region and $\rho\left(r=r_{L}\right)$ roughly that of the outer region. As shown in this table, regarding the same example in table 1, when the size of the system increases by one order of magnitude, the time increases by one order and the energy density decreases by two orders of magnitude. For this example to be consistent with the situation of our present universe, the time $t$ should be $\sim 10^{10}$ years and the energy density of the outer region should be similar to the average energy density of the present universe, $\sim 10^{-29} \mathrm{~g} \mathrm{~cm}^{-3}$. One can see that these two conditions cannot be simultaneously satisfied in this example, and therefore this example by itself alone cannot describe the present universe.

To study the dependence of the deceleration parameter $q_{r}$ on the six parameters in equation (35), we use the example in table 1 as a reference and tune one of the six parameters at

\footnotetext{
4 Note that in the inner region around the origin $r=0$ the energy density distribution is flat and therefore there is no singularity and, moreover, no cusp behavior (or weak singularity) in this acceleration example.

5 For a proof, see appendix A. The result is in equation (A.11).
} 

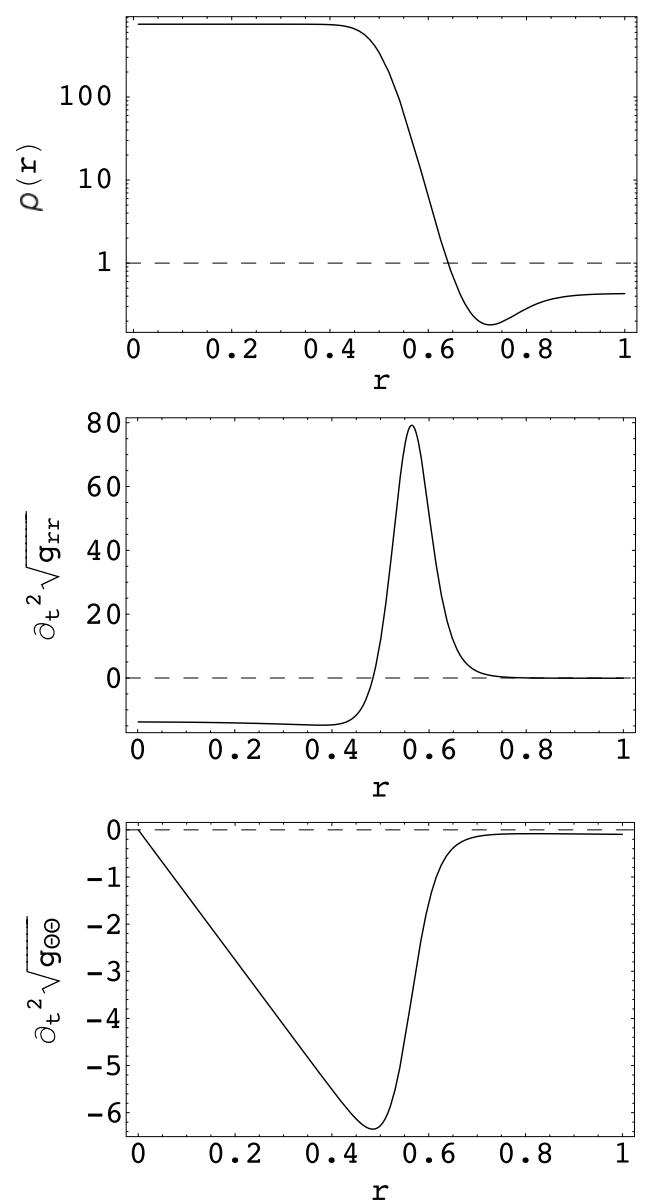

Figure 2. The plots of the physical energy density $\rho$ and the quantities, $\partial_{t}^{2} \sqrt{g_{r r}}$ and $\partial_{t}^{2} \sqrt{g_{\theta \theta}}$, which characterize the local acceleration/deceleration status in the radial and the angular direction, respectively, for the example in table 1 . We note that when $r=r_{L}=1, \partial_{t}^{2} \sqrt{g_{r r}}=-0.066$ and $\partial_{t}^{2} \sqrt{g_{\theta \theta}}=-0.095$.

Table 2. Corresponding to different length units, the values of several dimensionful quantities for the acceleration example in table 1 are presented.

\begin{tabular}{lllll}
\hline $\begin{array}{l}\text { Length unit } \\
(\mathrm{Mpc})\end{array}$ & $L_{r}(\mathrm{Mpc})$ & $t$ (year) & $\rho(r=0)\left(\mathrm{g} \mathrm{cm}^{-3}\right)$ & $\rho\left(r=r_{L}\right)\left(\mathrm{g} \mathrm{cm}^{-3}\right)$ \\
\hline 0.1 & 0.129 & $3.26 \times 10^{5}$ & $1.19 \times 10^{-29}$ & $6.80 \times 10^{-33}$ \\
1 & 1.29 & $3.26 \times 10^{6}$ & $1.19 \times 10^{-31}$ & $6.80 \times 10^{-35}$ \\
10 & 12.9 & $3.26 \times 10^{7}$ & $1.19 \times 10^{-33}$ & $6.80 \times 10^{-37}$ \\
100 & 129 & $3.26 \times 10^{8}$ & $1.19 \times 10^{-35}$ & $6.80 \times 10^{-39}$ \\
\hline
\end{tabular}

one time, while keeping the other five unchanged (i.e., with the values in table 1). The results are shown in figure 3 . The plot of $q_{r}$ versus $n_{k}$ shows that we have larger acceleration for larger $n_{k}$ that corresponds to larger inhomogeneity. This result again supports the possibility of the 

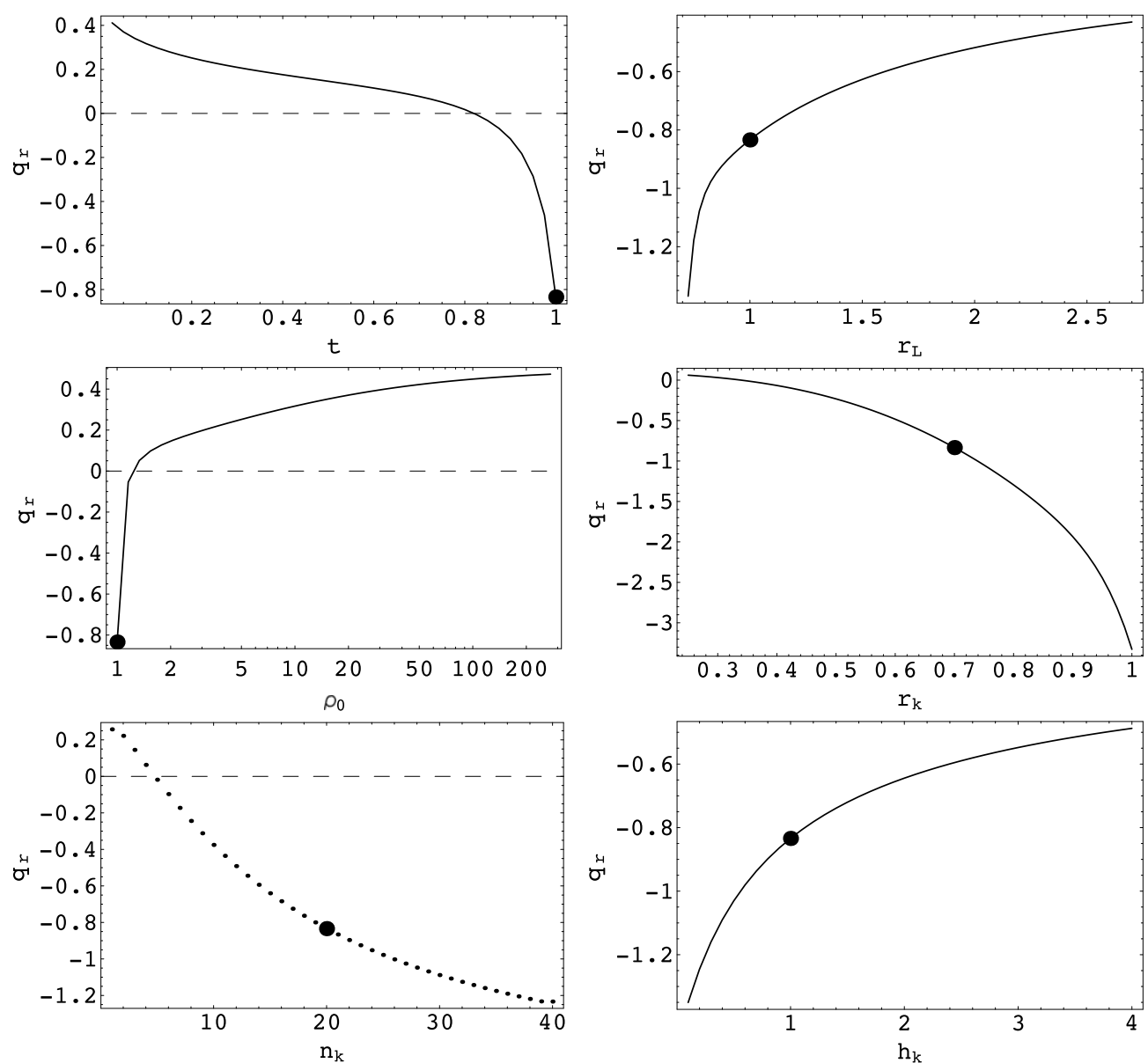

Figure 3. Illustration of the dependence of the deceleration parameter $q_{r}$ on the parameters $\left(t, r_{L}, \rho_{0}, r_{k}, n_{k}, h_{k}\right)$, using the example in table 1 as a reference (denoted by the large dot).

inhomogeneity-induced acceleration, in which, naively, inducing larger acceleration requires larger inhomogeneity. In addition, we have acceleration even for a moderate power $n_{k}$ (e.g., $n_{k}=5$ ), that is, for the purpose of generating the line acceleration we do not need very large inhomogeneity.

\section{Domain acceleration induced by inhomogeneities}

In this section, we investigate the domain acceleration for a spherical domain, $0<r<r_{D}$, with the volume

$$
V_{D}=4 \pi \int_{0}^{r_{D}} \frac{R^{2} R,{ }_{r}}{\sqrt{1+2 E(r)}} \mathrm{d} r=4 \pi \int_{0}^{r_{D}} \frac{a^{2} r^{2}\left(a+a,{ }_{r} r\right)}{\sqrt{1-k(r) r^{2}}} \mathrm{~d} r .
$$

The length invoked for the domain acceleration is that in equation (7): $L_{D}=V_{D}^{1 / 3}$, via which the deceleration parameter $q_{D}$ of the domain is defined in equation (9): $q_{D}=-\ddot{L}_{D} L_{D} / \dot{L}_{D}^{2}$. 
We note that in the case where $k(r)=0=E(r)$ there is no domain acceleration (see appendix B and [48]).

For the domain acceleration Kai et al [47] found examples based on the LTB solution, where constant $\rho_{0}(r)$, trivial $t_{b}(r)$ and the following $k(r)$ function are invoked:

$$
k(r)= \begin{cases}\frac{k_{0}}{2 r^{2}}\left\{\frac{\left(r^{2}-r_{2}^{2}\right)^{2}}{r_{1}^{2}-r_{2}^{2}}+r_{1}^{2}+r_{2}^{2}\right\} & \text { for } 0 \leqslant r<r_{1}, \\ \frac{k_{0}}{2 r^{2}}\left(r_{1}^{2}+r_{2}^{2}\right) & \text { for } r_{1} \leqslant r<r_{2}, \\ \frac{k_{0}}{2 r^{2}}\left(r_{1}^{2}+r_{2}^{2}\right)\left\{\left(\frac{r^{2}-r_{3}^{2}}{r_{\mathrm{b}}^{2}-r_{3}^{2}}\right)^{2}-1\right\}^{2} & \text { for } r_{2} \leqslant r<r_{3}, \\ 0 & \text { for } r_{3} \leqslant r<r_{\mathrm{b}}, \\ & \text { for } r_{\mathrm{b}} \leqslant r,\end{cases}
$$

where $0<r_{1}<r_{2}<r_{3}<r_{\mathrm{b}}$ and $k_{0}$ is a constant. In these examples [47], the acceleration involves the existence of a singularity around the origin. In contrast, we find the domain acceleration examples without singularity. The difference in the choice of the three functions which specify the LTB solution is that in our examples non-trivial $t_{b}(r)$ is invoked, as will be presented in the following. Our examples indicate that the existence of a singularity is not necessary for generating the domain acceleration.

In search of examples of the domain acceleration without singularity, we first follow the same procedures used in the previous section for the line acceleration and choose the functions, $k(r), \rho_{0}(r)$ and $t_{b}(r)$, as those in equations (32)-(34). In this case, we surveyed the six-dimensional parameter space $\left(t, r_{L}, \rho_{0}, r_{k}, n_{k}, h_{k}\right)$ and found no domain acceleration.

Contradicting our result, in [49] it was claimed that the example of the domain acceleration was found with constant $\rho_{0}$, trivial $t_{b}(r)$ and step-function-like $k(r)$ (i.e., with infinitely large $n_{k}$ in equation (32)). There is a mistake in the calculations of the volume $V_{D}$ in [49], where the authors ignored the volume at the transition point $r=r_{k}$ that is actually nonzero and should not be ignored (even though $r=r_{k}$ corresponds to a '2D surface' in the coordinate space). After taking the volume at $r=r_{k}$ back into account, we found no domain acceleration. (For more details, see appendix C.)

Since no domain acceleration (without singularity) was found in our search with trivial $t_{b}(r)$, we then consider a non-trivial function for $t_{b}(r)$,

$$
t_{b}(r)=-\frac{h_{t b}\left(r / r_{t}\right)^{n_{t}}}{1+\left(r / r_{t}\right)^{n_{t}}}
$$

while invoking the same functions for $k(r)$ and $\rho_{0}(r)$ in equations (32) and (33),

$$
\begin{aligned}
& k(r)=-\frac{\left(h_{k}+1\right)\left(r / r_{k}\right)^{n_{k}}}{1+\left(r / r_{k}\right)^{n_{k}}}+1, \\
& \rho_{0}(r)=\text { constant. }
\end{aligned}
$$

The behavior of the function $t_{b}(r)$ in equation (38) is illustrated in figure 4 .

With the above choice of the three functions involved in the LTB solution, we have nine free parameters to tune,

$$
\left(t, r_{D}, \rho_{0}, r_{k}, n_{k}, h_{k}, r_{t}, n_{t}, h_{t b}\right) .
$$

We surveyed this nine-dimensional parameter space and did find examples of the domain acceleration eventually. In table 3 , we present three examples with significantly different magnitude in acceleration (i.e., regarding the value of $q_{D}$ ). 


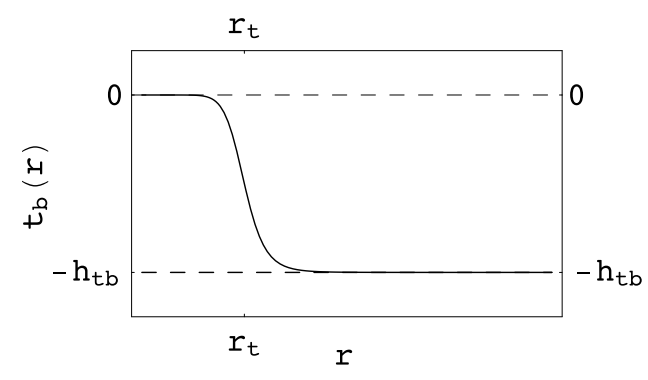

Figure 4. The plot of the function $t_{b}(r)$ invoked in the search for the domain acceleration.

Table 3. Three examples of the domain acceleration.

\begin{tabular}{rllllrrrrrr}
\hline & $t$ & $r_{D}$ & $\rho_{0}$ & \multicolumn{1}{c}{$r_{k}$} & \multicolumn{1}{c}{$n_{k}$} & \multicolumn{1}{c}{$h_{k}$} & $r_{t}$ & $n_{t}$ & $h_{t b}$ & $q_{D}$ \\
\hline 1 & 0.1 & 1 & 1 & 0.6 & 20 & 10 & 0.6 & 20 & 10 & -0.01 \\
2 & 0.1 & 1.1 & $10^{5}$ & 0.9 & 40 & 40 & 0.9 & 40 & 10 & -1.08 \\
3 & $10^{-8}$ & 1 & $10^{10}$ & 0.77 & 100 & 100 & 0.92 & 100 & 50 & -6.35 \\
\hline & $L_{D}$ & $\dot{L}_{D}$ & $\ddot{L}_{D}$ & $q_{D}$ & & & & & & \\
\hline 1 & 16.2 & 1.62 & 0.00174 & -0.01 & & & & & & \\
2 & 94.0 & 7.63 & 0.694 & -1.08 & & & & & & \\
3 & 8720 & 117 & 10.0 & -6.35 & & & & & & \\
\hline
\end{tabular}

The further details of these three examples are respectively illustrated in figures 5-7, which present the energy density distribution and the acceleration/deceleration status of the radial and the angular line elements. Similar to the example of the line acceleration in the previous section, in all these three examples the spherically symmetric dust fluid consists of two roughly homogeneous regions and one inhomogeneous transition/junction region ${ }^{6}$. Regarding the line elements, we get acceleration in the radial direction in the inhomogeneous region. How the evolution of the line elements affect the evolution of $L_{D}$ is not clear. Naively, it is a reasonable possibility that the domain acceleration stems from the acceleration of the radial line elements in the inhomogeneous region, that is, the existence of the accelerating line elements might be a necessary condition for the domain acceleration. Is it possible to have the domain acceleration without the acceleration of line elements? So far we do not have a no-go theorem prohibiting this possibility. However, we found no such example through our survey of the nine-dimensional parameter space. This might be an indication of the correlation between the domain acceleration and the acceleration of line elements.

For a demonstration of the scales of the size and other quantities of this system, in table 4 we fix the only one unspecified unit by using the length unit: $0.1 \mathrm{Mpc}, 1 \mathrm{Mpc}$, $10 \mathrm{Mpc}$ and $100 \mathrm{Mpc}$, and present the values of several dimensionful quantities (in addition to the dimensionless $q_{D}$ ), respectively. For each example in table 3, when the size of the system increases by one order of magnitude, the time increases by one order and the energy density decreases by two orders of magnitude. From this table one can see that the time $t$ and the energy density of the outer region cannot be simultaneously consistent with the situation of the present universe (i.e., $t_{0} \sim 10^{10}$ years and $\rho_{0} \sim 10^{-29} \mathrm{~g} \mathrm{~cm}^{-3}$ ), and therefore these three

6 Note that in these three acceleration examples the energy density distribution is flat around the origin $r=0$ and therefore there is no singularity and, moreover, no cusp behavior (or weak singularity). 

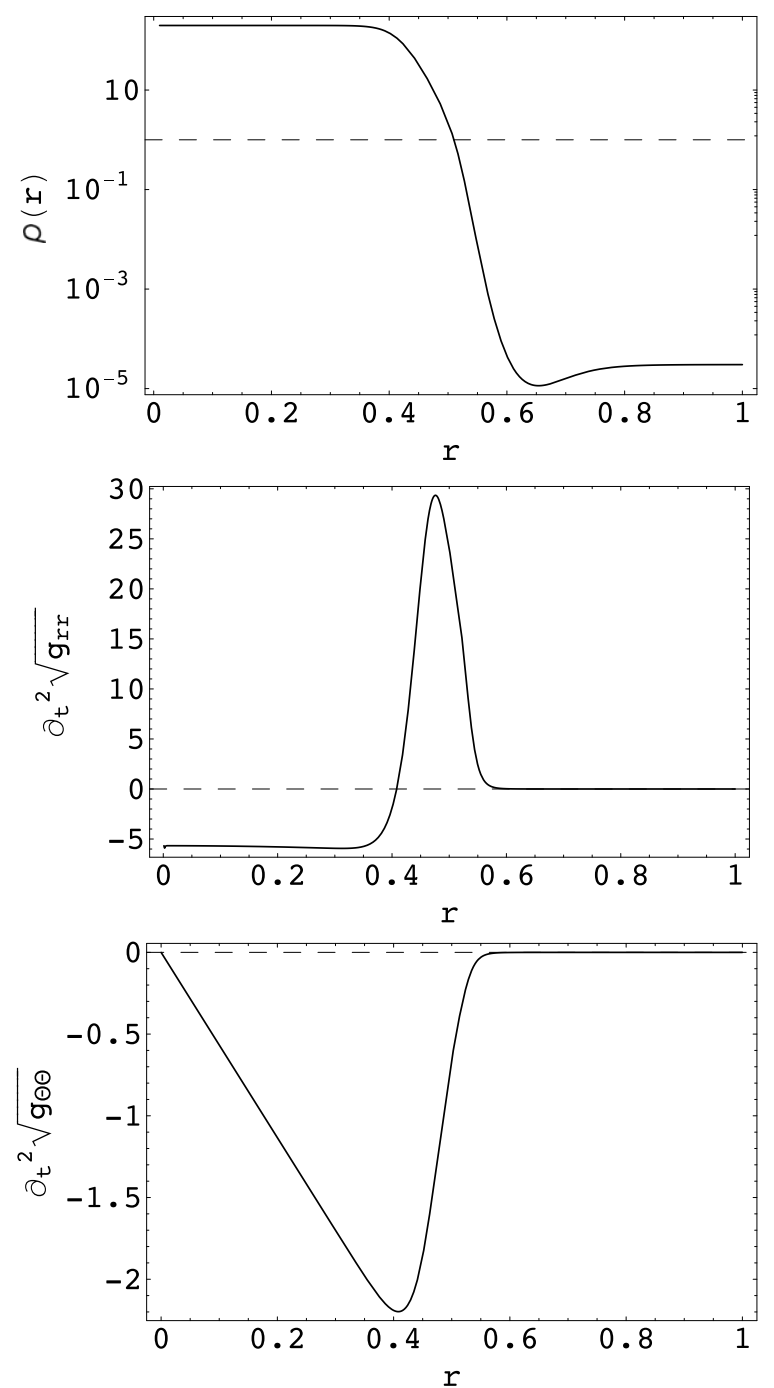

Figure 5. The plots of the physical energy density $\rho$ and the quantities, $\partial_{t}^{2} \sqrt{g_{r r}}$ and $\partial_{t}^{2} \sqrt{g_{\theta \theta}}$, which characterize the local acceleration/deceleration status in the radial and the angular direction, respectively, for the first example in table 3 . Note that when $r=r_{D}=1, \partial_{t}^{2} \sqrt{g_{r r}}=-4.9 \times 10^{-5}$ and $\partial_{t}^{2} \sqrt{g_{\theta \theta}}=-1.6 \times 10^{-4}$.

examples by themselves cannot describe the present universe. The search (based on the LTB solution) of the domain acceleration examples which are consistent with observational results is important and worthy of further investigation.

To study the dependence of the deceleration parameter $q_{D}$ on the nine parameters in equation (41), we use the second example in table 3 as a reference and tune one of these nine parameters at one time, while keeping the other eight unchanged (i.e., with the values in the second example in table 3). The results are shown in figure 8 . These plots show that every parameter has significant influence on $q_{D}$ (at least within some range of the value of the parameter). We note that in the case of the inhomogeneous function $t_{b}(r)$ in equation (38) we 

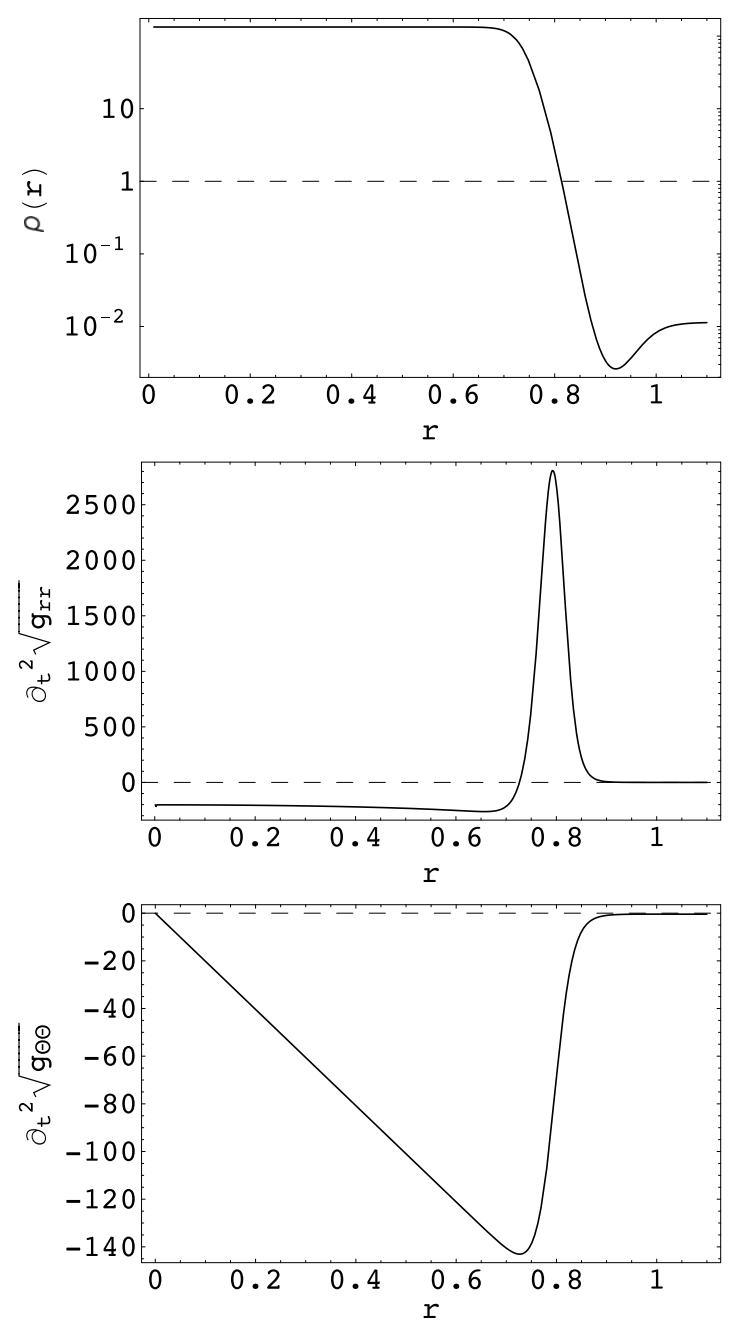

Figure 6. The plots of the physical energy density $\rho$ and the quantities, $\partial_{t}^{2} \sqrt{g_{r r}}$ and $\partial_{t}^{2} \sqrt{g_{\theta \theta}}$, which characterize the local acceleration/deceleration status in the radial and the angular direction, respectively, for the second example in table 3. Note that when $r=r_{D}=1.1, \partial_{t}^{2} \sqrt{g_{r r}}=-0.055$ and $\partial_{t}^{2} \sqrt{g_{\theta \theta}}=-0.43$.

have acceleration or deceleration in different situations with different values of parameters, and, moreover, the examples of the domain acceleration are not rare. Conversely, in the case of trivial $t_{b}(r)$ we found no domain acceleration without singularity. Accordingly, in the LTB solution the function $t_{b}(r)$ plays a key role in generating the domain acceleration.

\section{Summary, discussions and outlook}

Against the common consensus (in equation (1)) that normal matter always slows down the expansion of the universe, we have found and demonstrated examples of the line acceleration and the domain acceleration for a spherically symmetric dust fluid described by the LTB solution without singularity. This discovery contradicts the common intuition about the interplay of gravity and the cosmic evolution. Furthermore, these examples have shown the 

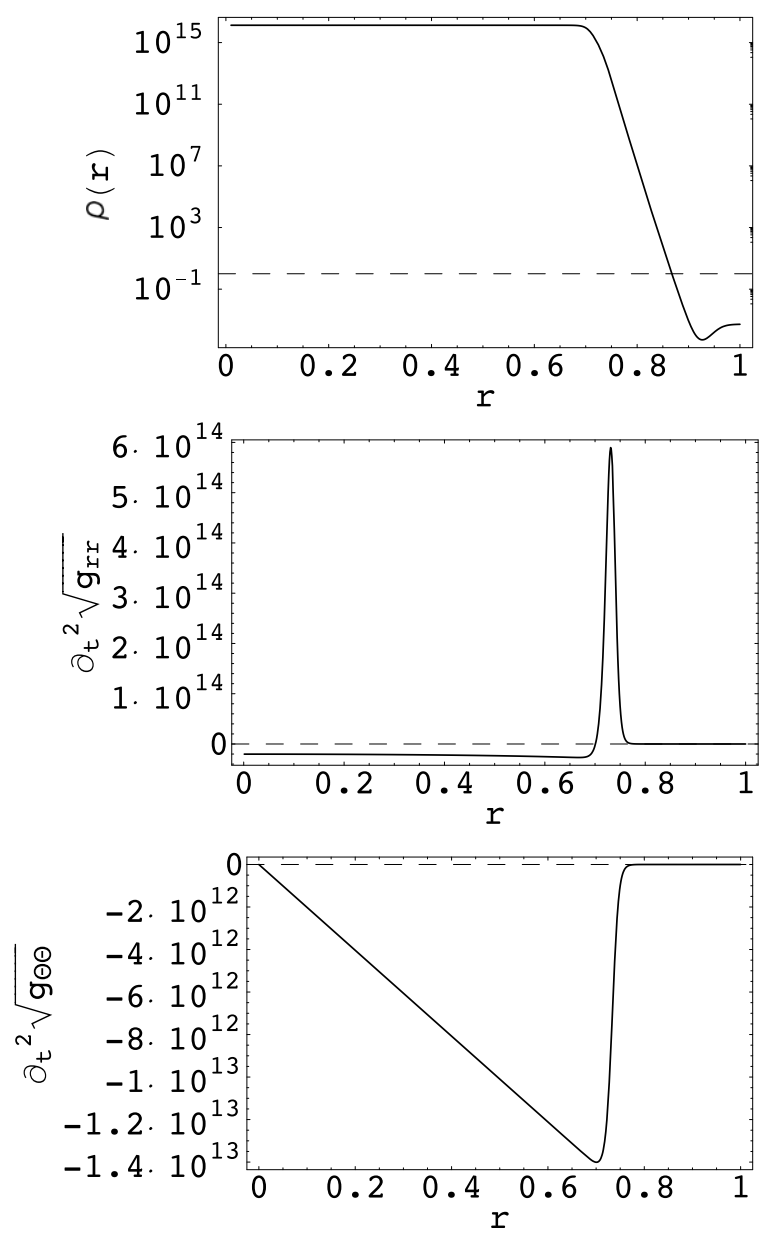

Figure 7. The plots of the physical energy density $\rho$ and the quantities, $\partial_{t}^{2} \sqrt{g_{r r}}$ and $\partial_{t}^{2} \sqrt{g_{\theta \theta}}$, which characterize the local acceleration/deceleration status in the radial and the angular direction, respectively, for the third example in table 3. Note that when $r=r_{D}=1, \partial_{t}^{2} \sqrt{g_{r r}}=-0.23$ and $\partial_{t}^{2} \sqrt{g_{\theta \theta}}=-2.36$.

strong correlation between acceleration and inhomogeneity. These results strongly support the suggestion that inhomogeneity can induce acceleration.

In these acceleration examples the spherically symmetric dust fluid consists of three regions: two (roughly) homogeneous regions - the inner over-density region and the outer under-density region - and one transition/junction region, where the inhomogeneity locates, of two homogeneous regions. Note that in these examples the energy density distribution is flat around the origin $r=0$ and therefore there is no singularity and, moreover, no cusp behavior (or weak singularity).

For further understanding the acceleration, the quantity $\partial_{t}^{2} \sqrt{g_{r r}}$, which characterizes the acceleration/deceleration status of an infinitesimal radial line element, is one of the good quantities to study. Naively, the regions with positive/negative $\partial_{t}^{2} \sqrt{g_{r r}}$ make positive/negative contribution to acceleration. For the quantity $\partial_{t}^{2} \sqrt{g_{r r}}$ to be positive we find a necessary and sufficient condition, $\left(a^{2} / r\right)_{, r}>0$. This condition tells us that a positive contribution to 
Table 4. Corresponding to different length units, the values of several dimensionful quantities for the acceleration examples in table 3 are presented.

\begin{tabular}{|c|c|c|c|c|c|c|}
\hline & $q_{D}$ & $\begin{array}{l}\text { Length unit } \\
(\mathrm{Mpc})\end{array}$ & $t$ (year) & $L_{r}(\mathrm{Mpc})$ & $\rho(r=0)\left(\mathrm{g} \mathrm{cm}^{-3}\right)$ & $\rho\left(r=r_{D}\right)\left(\mathrm{g} \mathrm{cm}^{-3}\right)$ \\
\hline \multirow[t]{4}{*}{1} & \multirow[t]{4}{*}{-0.01} & 0.1 & $3.26 \times 10^{4}$ & 1.62 & $3.15 \times 10^{-30}$ & $4.81 \times 10^{-37}$ \\
\hline & & 1 & $3.26 \times 10^{5}$ & 16.2 & $3.15 \times 10^{-32}$ & $4.81 \times 10^{-39}$ \\
\hline & & 10 & $3.26 \times 10^{6}$ & 162 & $3.15 \times 10^{-34}$ & $4.81 \times 10^{-41}$ \\
\hline & & 100 & $3.26 \times 10^{7}$ & 1620 & $3.15 \times 10^{-36}$ & $4.81 \times 10^{-43}$ \\
\hline \multirow[t]{4}{*}{2} & \multirow[t]{4}{*}{-1.08} & 0.1 & $3.26 \times 10^{4}$ & 9.4 & $2.11 \times 10^{-30}$ & $1.79 \times 10^{-34}$ \\
\hline & & 1 & $3.26 \times 10^{5}$ & 94 & $2.11 \times 10^{-32}$ & $1.79 \times 10^{-36}$ \\
\hline & & 10 & $3.26 \times 10^{6}$ & 940 & $2.11 \times 10^{-34}$ & $1.79 \times 10^{-38}$ \\
\hline & & 100 & $3.26 \times 10^{7}$ & 9400 & $2.11 \times 10^{-36}$ & $1.79 \times 10^{-40}$ \\
\hline \multirow[t]{4}{*}{3} & \multirow[t]{4}{*}{-6.35} & 0.1 & $3.26 \times 10^{-3}$ & 872 & $2.11 \times 10^{-16}$ & $8.33 \times 10^{-36}$ \\
\hline & & 1 & $3.26 \times 10^{-2}$ & 8720 & $2.11 \times 10^{-18}$ & $8.33 \times 10^{-38}$ \\
\hline & & 10 & $3.26 \times 10^{-1}$ & 87200 & $2.11 \times 10^{-20}$ & $8.33 \times 10^{-40}$ \\
\hline & & 100 & 3.26 & 872000 & $2.11 \times 10^{-22}$ & $8.33 \times 10^{-42}$ \\
\hline
\end{tabular}

acceleration is made in the place where $a(t, r)$ increases sufficiently fast with the radial coordinate $r$. In every acceleration example we found, there exists a region with positive $\partial_{t}^{2} \sqrt{g_{r r}}$ that coincides with the inhomogeneous transition/junction region quite well. This result reveals the strong correlation between acceleration and inhomogeneity, thereby giving a strong support to the suggestion that inhomogeneity can induce accelerating expansion.

It is not clear how the line acceleration and domain acceleration are related to the apparent acceleration. A similar doubt (regarding the domain acceleration) was also raised by Kai et al in [47]. The relation between the theoretical accelerations-line acceleration and domain acceleration-and the apparent acceleration could be model dependent. For our universe with complicated energy distribution the relation may be far from simple. As a reminder, we note that the conclusion about the existence of the present cosmic acceleration indicated by observations is based on the homogeneous and isotropic FLRW cosmology. If one invokes inhomogeneous cosmology, the current observational results may not indicate the existence of the cosmic acceleration. As shown in appendix B and [48], in the cases where $E(r)=0$ there is no acceleration (for both the line and the domain acceleration). In contrast, it has been shown that it is possible to fit supernova data in the LTB model with trivial $E(r)$ [26]. This gives an example of having apparent acceleration while having no line acceleration and no domain acceleration. One may treat a complicated universe as a large domain consisting of many different sub-domains, and from a statistical perspective it might be reasonable that the size $L_{D}$ of the large domain corresponds to the scale factor in the FRW metric. In many works the results from the analysis involving the quantity $L_{D}$ are compared with observations in this spirit $[36,50,42,51,60,37]$. Nevertheless, whether this is a good approximation is not clear yet.

One may wonder whether the acceleration in our examples is real or fake, i.e., whether it truly corresponds to the space expansion. One example leading to this concern is to consider an accelerating system consisting of two decelerating regions. Since each region is decelerating one might expect that the domain acceleration in this case does not correspond to physically observable attributes (e.g. the luminosity distance-redshift relation) of an accelerating FLRW model [39]. Nevertheless, in contrast, it has been shown that for a system consisting of decelerating regions it is possible to fit the supernova data or to generate apparent acceleration $[26,43]$. 

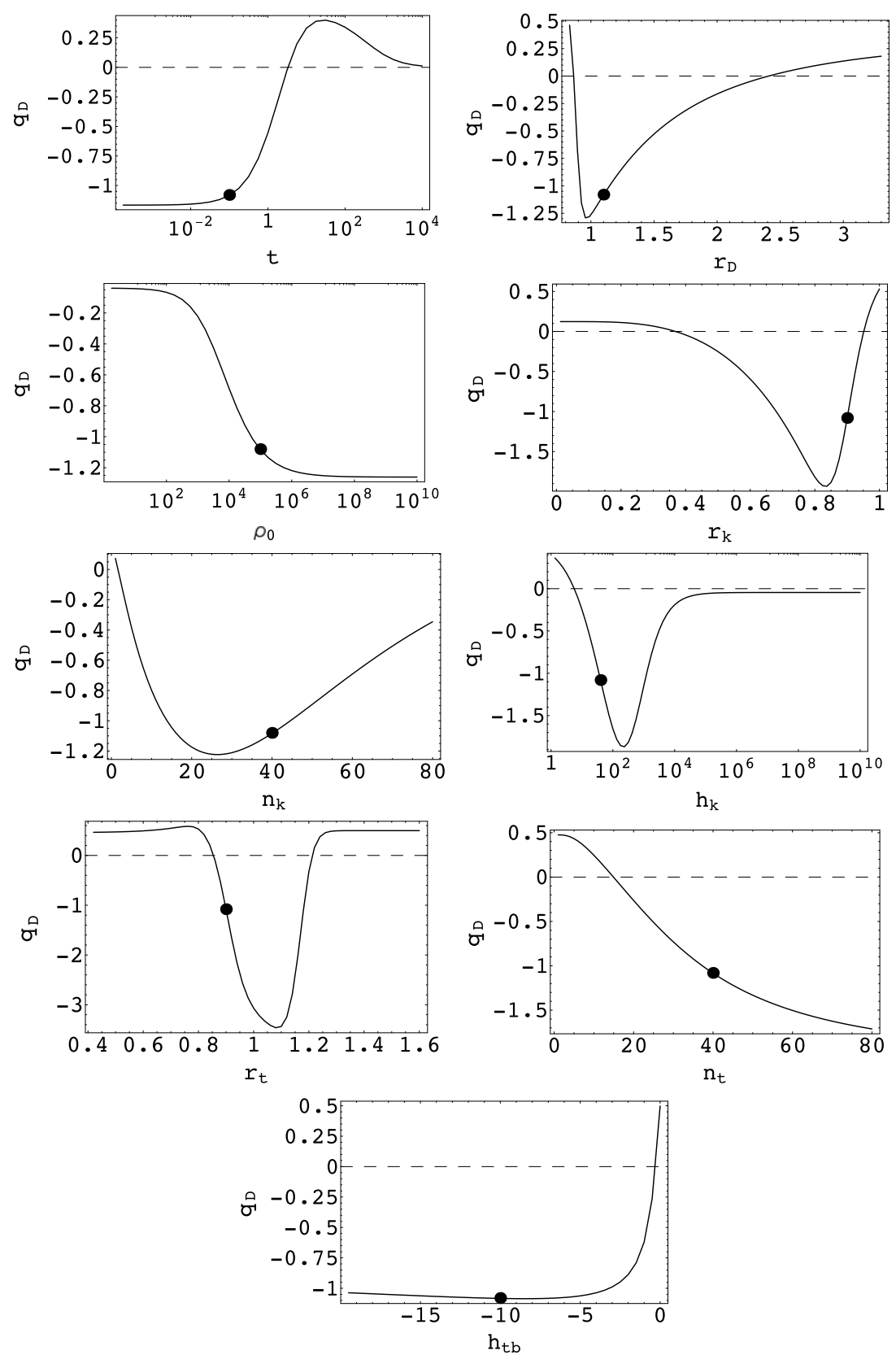

Figure 8. Illustration of the dependence of the deceleration parameter $q_{D}$ on the parameters $\left(t, r_{D}, \rho_{0}, r_{k}, n_{k}, h_{k}, r_{t}, n_{t}, h_{t b}\right)$, using the second example in table 3 as a reference (denoted by the large dot).

In addition, as already pointed out in the introduction, it needs much caution to connect separate regions or to put them together. Two particular important issues (that may be closely related to each other) are: 
(1) There may be singularity in the junction between separate regions.

(2) The effect from the junction between separate regions may be significant and should be taken into account seriously.

We have already had a lesson from the work by Nambu and Tanimoto in [49], where they basically considered a system (of spherical symmetry) consisting of an inner and an outer decelerating FLRW region and, as a result, they found and presented examples of the domain acceleration. In this system there is singularity in the junction between these two regions, and in their treatment the contribution from the junction is ignored. As pointed out in the previous section (with details in appendix C), it is inappropriate to ignore the junction. Actually the junction makes a significant contribution to the volume of the system, although it looks like a '2D' surface in the coordinate space. After taking care of the singularity and taking into account the volume of the junction, we find that there exists no domain acceleration in the cases studied in [49].

Another concern is about the definition of acceleration, as discussed in section 2. The two definitions employed in the present paper-line acceleration and domain acceleration, on which our acceleration examples are based-follow the scenario proposed in section 2 for a system of freely moving particles interacting with each other only through gravity. For the purpose of truly representing the space evolution status while avoiding the confusion from particle motion and fake frame acceleration, the length quantities invoked in this scenario to define acceleration are (1) the distance between two freely moving particles for the line acceleration and (2) the size of a spatial region with a constant number of particles therein for the domain acceleration. Regarding the frame/gauge choice, we have emphasized the advantage of the synchronous gauge in which the above requirements for the length involved in the acceleration definition can be easily met. In addition, in this gauge, there exists a universal cosmic time that is the proper time of comoving observers, and for a region with its boundary fixed in the coordinate space the volume expansion rate coincides with the expansion rate of the physical proper volume.

The counter-intuitive acceleration examples we found raise two issues worthy of further investigations:

- How to understand these counter-intuitive examples?

- Can inhomogeneities explain 'cosmic acceleration'?

Regarding the first issue, the common intuition about equation (1) may actually stem from Newtonian gravity that is a perturbed version of general relativity in the Newtonian limit with the Minkowski spacetime as the background. Accordingly, this intuition may be valid only for considering the particle motion relative to a background spacetime in a perturbative framework, but may be invalid for considering the evolution of spacetime that is described by general relativity. For further understanding the cosmic evolution and other topics involving the general-relativity effects as the dominant effects, it will be helpful if one can build the intuition about general relativity, i.e., with which one can make a proper guess at the behavior of the spacetime geometry for an arbitrarily given energy distribution.

Regarding the second issue, the most important is whether the inhomogeneities of our universe can explain the supernova data. If yes, the next step is to see, according to observational data, how the universe evolves, in particular, whether the cosmic acceleration exists or not, in this cosmological model taking the real inhomogeneities into account. So far the examples we found may be far away from the real situation of our universe. How to benefit from these mathematical examples in order to understand the present cosmic acceleration through the inhomogeneities of our universe is an important issue currently under our investigation. It doesn't matter whether the cosmic acceleration can eventually 
be explained simply by inhomogeneity, these examples have shown that inhomogeneity may affect the cosmic evolution in a manner far beyond the usual naive intuition about the interplay of gravity and the spacetime geometry. Thus, the effects of inhomogeneity on the cosmic evolution should be restudied carefully. These examples open a new perspective on the understanding of the evolution of our universe.

\section{Acknowledgments}

We thank P-M Ho for useful discussions. This work was supported by the National Science Council, Taiwan, ROC (NSC 94-2752-M-002-007-PAE, NSC 94-2112-M-002-029, NSC 942811-M-002-048, NSC 94-2112-M-002-036 and NSC 93-2112-M-002-009). Gu also thanks the National Center for Theoretical Sciences (funded by the National Science Council), Taiwan, ROC for the support.

\section{Appendix A. Restrictions on the LTB solution}

In this appendix, we will give several reasonable restrictions on the LTB solution and study the properties of the LTB solution under these restrictions. We consider three conditions: (1) no hole, (2) no singularity and (3) non-negative and finite energy density. For no hole at the center, the area of the spherical surface at $r=r_{0}$ should go to zero when $r_{0}$ goes to zero. Accordingly, condition (1) requires

$$
R(t, r=0)=0 .
$$

Condition (2) requires the smooth behavior of the functions involved in the LTB solution. In particular, we consider

$$
R, r(t, r) \neq 0
$$

Condition (3) requires

$$
0 \leqslant \rho(t, r)<\infty
$$

Note that the necessary and sufficient conditions of no shell crossing in a period of time are presented by Hellaby and Lake in [59]. The conditions discussed here are only necessary conditions.

Because $R,{ }_{r}$ is nonvanishing and continuous, $R,_{r}$ cannot change its sign, i.e., $R, r$ is either always positive or always negative for all $r$. Because $R(t, r=0)=0$, the $\operatorname{sign}$ of $R(t, r>0)$ should be the same as that of $R,{ }_{r}$, i.e.,

$$
R R, r>0 \quad \text { for } \quad r>0 .
$$

This property will be used in appendix $\mathrm{C}$ regarding the possibility of generating the domain acceleration with trivial $t_{b}(r)$.

Considering equation (13),

$$
\left(\frac{\dot{R}}{R}\right)^{2}=\frac{2 E(r)}{R^{2}}+\frac{2 M(r)}{R^{3}},
$$

for every term therein to be finite, we obtain

$$
\begin{aligned}
& E(r=0)=0, \\
& M(r=0)=0,
\end{aligned}
$$


because $R(t, r=0)=0$. From equation (14),

$$
\rho(t, r)=\frac{2 M^{\prime}(r)}{R^{2} R, r},
$$

equation (A.3) requires

$$
\frac{M^{\prime}(r)}{R, r(t, r)} \geqslant 0 \text {. }
$$

Because $R,{ }_{r}$ cannot change its sign, $M^{\prime}(r)$ cannot change its sign either according to the above relation. Using the same reasoning for obtaining equation (A.4), from equation (A.6) we have

$$
M(r) M^{\prime}(r) \geqslant 0 \text {. }
$$

To sum up, so far we have obtained the following basic restrictions on the LTB solution: $R(t, r=0)=E(r=0)=M(r=0)=0 ; R, R, r, M(r)$ and $M^{\prime}(r)$ have the same sign when nonvanishing.

Examining equations (13) and (14), we can see the feature that for a solution where $R(t, r) \leqslant 0$ there is always another solution $R_{1}(t, r) \geqslant 0$, obeying

$$
R_{1}(t, r)=-R(t, r), \quad M_{1}(r)=-M(r), \quad E_{1}(r)=E(r) .
$$

Thus, without losing generality, we can set

$$
R(t, r) \geqslant 0, \quad R,_{r}>0, \quad M(r) \geqslant 0, \quad M^{\prime}(r) \geqslant 0 .
$$

With this choice, regarding the dependence on the radial coordinate $r, R$ is a monotonically increasing function and $M$ is a non-decreasing function, while both vanish at the origin $r=0$.

In the following, we will study $\ddot{R}(t, r)$ and show that $\ddot{R}(t, r) \leqslant 0$ and $\ddot{R}(t, r=0)=0$ corresponding to the choice in equation (A.10). The time derivative of equation (13) multiplied by $R^{2}$ gives a simple formula for $\ddot{R}$,

$$
\ddot{R}=-\frac{M(r)}{R^{2}}<0,
$$

corresponding to the choice in equation (A.10). According to equations (A.1) and (A.6) and applying L'Hospital's rule, we have

$$
\ddot{R}(t, r=0)=-\left.\frac{M}{R^{2}}\right|_{r=0}=-\left.\frac{M^{\prime}}{2 R R,_{r}}\right|_{r=0}=-\left.\frac{1}{4} R \rho\right|_{r=0},
$$

where equation (14) has been applied in the last equality. As a result, for a finite physical energy density $\rho$,

$$
\ddot{R}(t, r=0)=0,
$$

because $R(t, r=0)=0$.

\section{Appendix B. No acceleration with $E(r)=0=k(r)$}

In this appendix we consider the trivial case, $E(r)=0=k(r)$, and prove that there is no line acceleration and no domain acceleration in this case. (For the case of domain acceleration, see also [48].) 


\section{Line acceleration}

In the case with trivial $E(r)$ or $k(r)$, equations (29) and (30) give

$$
L_{r}(t) \equiv \int_{0}^{r_{L}} \sqrt{g_{r r}} \mathrm{~d} r=\int_{0}^{r_{L}} R,{ }_{r} \mathrm{~d} r=R\left(t, r_{L}\right),
$$

where equation (A.1), $R(t, r=0)=0$, has been used, and therefore

$$
q_{r} \equiv-\frac{\ddot{L}_{r} L_{r}}{\dot{L}_{r}^{2}}=-\left.\frac{\ddot{R} R}{\dot{R}^{2}}\right|_{r=r_{L}} .
$$

From equation (13), we have

$$
\dot{R}^{2}=\frac{2 M(r)}{R}
$$

and

$$
\ddot{R}=-\frac{M(r)}{R^{2}} .
$$

Substituting these two equations into equation (B.2) gives a simple result

$$
q_{r}=\frac{1}{2} \text {. }
$$

Thus, in the case with trivial $E(r)$ or $k(r)$, no matter how we tune the other two functions $\rho_{0}(r)$ and $t_{b}(r)$, the deceleration parameter corresponding to the proper distance between the origin and any other point in space is always $1 / 2$, a positive constant denoting a deceleration as large as that in a homogeneous dust-dominated or pressureless-matter-dominated universe.

\section{Domain acceleration}

When $E(r)=0=k(r)$, equation (36) gives

$$
\begin{aligned}
& V_{D}=4 \pi \int_{0}^{r_{D}} R^{2} R, r \mathrm{~d} r=\frac{4 \pi}{3} R^{3}\left(t, r_{D}\right), \\
& L_{D} \equiv V_{D}^{1 / 3}=\left(\frac{4 \pi}{3}\right)^{1 / 3} R\left(t, r_{D}\right),
\end{aligned}
$$

and therefore

$$
q_{D} \equiv-\frac{\ddot{L}_{D} L_{D}}{\dot{L}_{D}^{2}}=-\left.\frac{\ddot{R} R}{\dot{R}^{2}}\right|_{r=r_{D}}=\frac{1}{2},
$$

where equations (B.3) and (B.4) have been applied in the last equality.

Thus, the deceleration parameters corresponding to the domain acceleration and the line acceleration are both $1 / 2$, a positive constant (denoting deceleration) independent of the other two functions $\rho_{0}(r)$ and $t_{b}(r)$. This indicates that a non-trivial $E(r)$ or $k(r)$ function must play an essential role in the inhomogeneity-induced accelerating expansion based on the LTB solution.

\section{Appendix C. Domain acceleration with trivial $t_{b}(r)$ ?}

In [49] Nambu and Tanimoto claimed that the example of the domain acceleration was found. However, we find that it is an improper example. In this appendix we will study the example in [49] and discuss its problems. 


\section{C.1. Nambu and Tanimoto's example}

In [49] the choice of the three arbitrary functions involved in the LTB solution are specified as follows:

$$
\begin{aligned}
& \rho_{0}(r)=\rho_{0}=\text { constant }, \\
& t_{b}(r)=0, \\
& k(r)=\frac{1}{L^{2}}\left[2 \theta\left(r-r_{k}\right)-1\right], \quad 0 \leqslant r \leqslant L, \quad 0 \leqslant r_{k} \leqslant L,
\end{aligned}
$$

where $\theta(r)$ is a step function. In the following study, we will choose $L=1$ without losing generality, that is,

$$
k(r)=2 \theta\left(r-r_{k}\right)-1, \quad 0 \leqslant r \leqslant 1, \quad 0 \leqslant r_{k} \leqslant 1 .
$$

With the above choice the spherically symmetric dust fluid described by the LTB solution seems to consist of two regions described, respectively, by two different Robertson-Walker (RW) metrics: the inner region $\left(0 \leqslant r<r_{k}\right)$ described by a (spatially) open RW metric with the scale factor $a_{1}(t)$ and the outer region $\left(r_{k}<r \leqslant 1\right)$ described by a closed RW metric with the scale factor $a_{2}(t)$. The scale factors $a_{1}(t)$ and $a_{2}(t)$ obey the following Friedmann equations, respectively:

$$
\begin{aligned}
& \left(\frac{\dot{a}_{1}}{a_{1}}\right)^{2}=\frac{1}{a_{1}^{2}}+\frac{\rho_{0}}{3 a_{1}^{3}}, \\
& \left(\frac{\dot{a}_{2}}{a_{2}}\right)^{2}=-\frac{1}{a_{2}^{2}}+\frac{\rho_{0}}{3 a_{2}^{3}} .
\end{aligned}
$$

The initial condition

$$
a_{1}(t=0)=a_{2}(t=0)=0
$$

is used to solve the above equations and to obtain the time evolution of the scale factors $\left(a_{1}\right.$ and $a_{2}$ ) and accordingly the time evolution of the domain $\{0<r<L=1\}$.

To obtain the deceleration parameter $q_{D}$ of this domain, the following formulae for calculating the volume of the domain are used:

$$
\begin{aligned}
V_{D}(t) & =4 \pi \int_{0}^{1} \frac{a^{2} r^{2}\left(a+a,{ }_{r} r\right) \mathrm{d} r}{\sqrt{1-k(r) r^{2}}} \\
& =4 \pi\left[c_{1} a_{1}^{3}(t)+c_{2} a_{2}^{3}(t)\right],
\end{aligned}
$$

where $c_{1}$ and $c_{2}$ are constants defined as follows:

$$
c_{1}=\int_{0}^{r_{k}} \frac{x^{2} \mathrm{~d} x}{\sqrt{1+x^{2}}}, \quad c_{2}=\int_{r_{k}}^{1} \frac{x^{2} \mathrm{~d} x}{\sqrt{1-x^{2}}} .
$$

This volume calculation (i.e., the total volume being equal to the sum of the volumes of the inner region and the outer region) looks reasonable, but actually is incorrect, as to be discussed later.

For demonstration, in the following we consider the special case with $\rho_{0}=3$. The result about the time evolution of $q_{D}$, obtained by using equations (C.5)-(C.9), is shown in figure $\mathrm{C} 1$. This figure illustrates the existence of the domain acceleration at later times for several different values of $r_{k}$. 


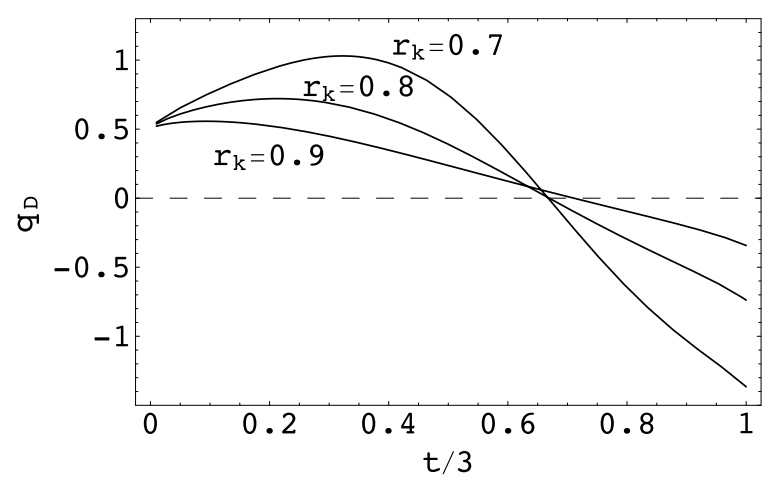

Figure C1. Evolution of the deceleration parameter $q_{D}$ of a spherically symmetric domain, which consists of an inner open RW region and an outer closed RW region, for $\rho_{0}=3$ and $r_{k}=0.7,0.8,0.9$.

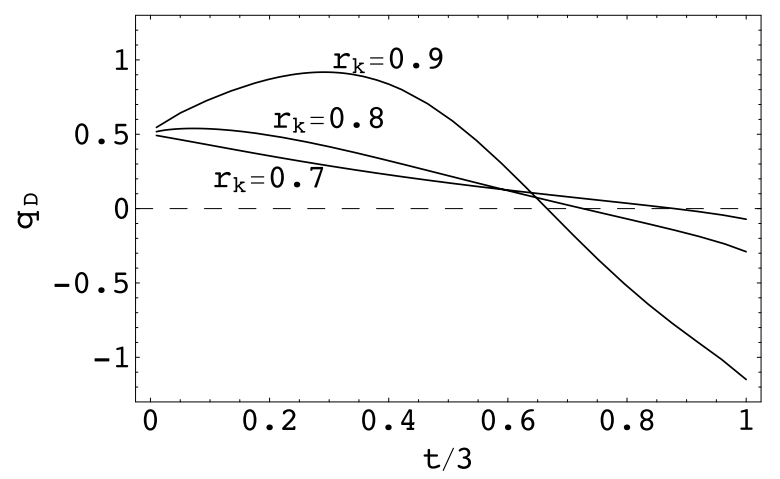

Figure C2. Evolution of the deceleration parameter $q_{D}$ of a spherically symmetric domain, which consists of an inner closed RW region and an outer open RW region, for $\rho_{0}=3$ and $r_{k}=0.7,0.8,0.9$.

Note that with the initial condition in equation (C.7) we have $a_{1}(t)>a_{2}(t)$ as $t>0$. This contradicts the restriction in equation (A.4) around $r=r_{k}$ (i.e., around the junction of two regions), and accordingly indicates the existence of a singularity that stems from the increasing behavior of $k(r)$ around $r_{k}$ (but is irrelevant to the singular behavior of $k(r)$ around $r_{k}$ ). This singularity can be avoided by exchanging the inner region and the outer region, making the function $k(r)$ decreasing instead of increasing, which is what we did about the choice of $k(r)$ in our search for acceleration examples.

Accordingly, with this change, $k(r)$ becomes

$$
k(r)=-2 \theta\left(r-r_{k}\right)+1, \quad 0 \leqslant r \leqslant 1, \quad 0 \leqslant r_{k} \leqslant 1 .
$$

In this case, the inner region $\left\{0 \leqslant r<r_{k}\right\}$ is described by the closed RW metric with the scale factor $a_{1}(t)$ and the outer region $\left\{r_{k}<r \leqslant 1\right\}$ by the open RW metric with $a_{2}(t)$, satisfying $a_{2}(t)>a_{1}(t)$ as $t>0$. The result about $q_{D}(t)$ for the case with above $k(r)$ and $\rho_{0}=3$ is demonstrated in figure $\mathrm{C} 2$.

As shown in figures $\mathrm{C} 1$ and $\mathrm{C} 2$, for both choices of $k(r)$, there exists the domain acceleration (at later times). However, as to be shown in the following section, after smoothing 


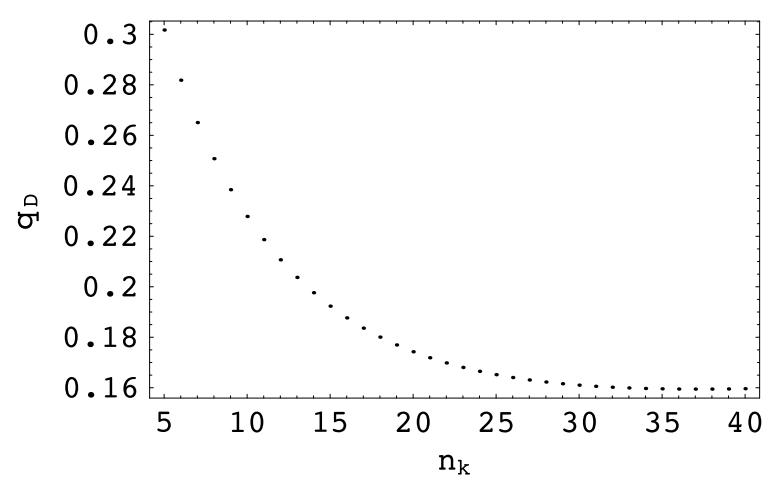

Figure C3. The dependence of the deceleration parameter $q_{D}$ on the power $n_{k}$ in the function $k(r)$ in equation (C.11) for $t=1, \rho_{0}=3$ and $r_{k}=0.9$.

the step function invoked in above $k(r)$ in order to get rid of the other singularity, we find no domain acceleration, no matter how close to that in equation (C.10) the function $k(r)$ is.

\section{C.2. Problems and revision}

In this section, we use the following function $k(r)$ to replace that involving a step function in the previous section:

$$
k(r)=-\frac{2\left(r / r_{k}\right)^{n_{k}}}{1+\left(r / r_{k}\right)^{n_{k}}}+1 .
$$

When the power $n_{k}$ goes to infinity, the above function $k(r)$ approaches to that in equation (C.10) involving a step function. If there is nothing wrong, we should obtain the same result about $q_{D}(t)$ for the case invoking above $k(r)$ with $n_{k} \rightarrow \infty$ and the case invoking $k(r)$ in equation (C.10). For demonstration we choose $t=1, \rho_{0}=3$ and $r_{k}=0.9$, corresponding to the case where $q_{D} \sim-1$ as shown in figure C2 for $k(r)$ in equation (C.10). In figure $\mathrm{C} 3$ the dependence of $q_{D}$ on $n_{k}$ for the case with $k(r)$ in equation (C.11) is illustrated. We can see that along with the increasing of the power $n_{k}$ the domain deceleration parameter $q_{D}$ always keeps far away from the value -1 (that corresponds to significant acceleration), and smoothly approaches to a positive value around 0.16 (deceleration).

Where does this discrepancy come from? The discrepancy stems from the doubtful calculation of the volume $V_{D}$ in [49] described in the previous section. In [49] Nambu and Tanimoto ignored the volume of the junction (between two RW regions) at $r=r_{k}$ that is actually significantly nonzero (even though $r=r_{k}$ corresponds to a '2D surface' in the coordinate space) and should not be ignored. After taking back the volume at $r=r_{k}$ into account as a more appropriate treatment, we found no domain acceleration in the cases discussed in [49].

In the following, we study the volume of the junction region around $r_{k}$ for the function $k(r)$ in equation (C.11),

$$
V_{\text {junction }}=4 \pi \int_{r_{k}-\varepsilon}^{r_{k}+\varepsilon} \frac{a^{2} r^{2}(a+a, r r) \mathrm{d} r}{\sqrt{1-k(r) r^{2}}},
$$

where

$$
\varepsilon=\frac{1}{2 n_{k}}
$$



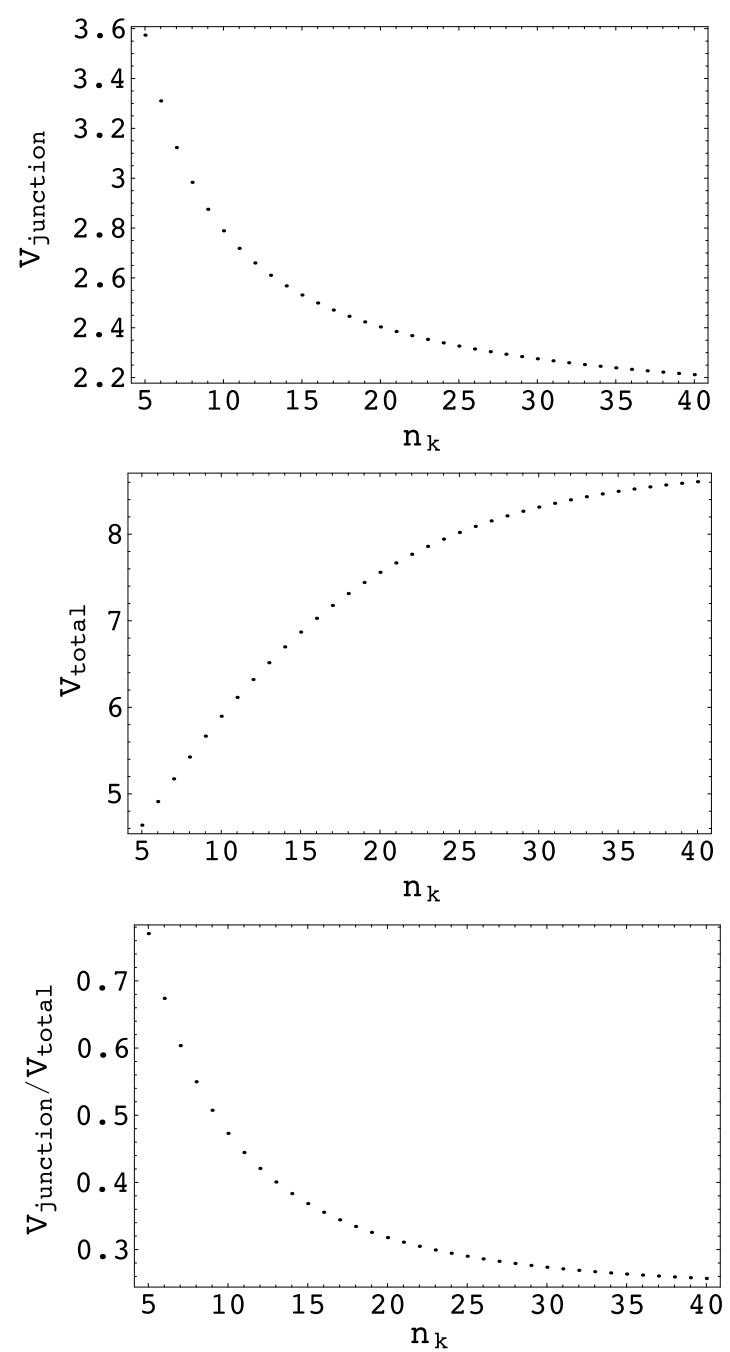

Figure C4. The dependence of the volume of the junction and the total volume on the power $n_{k}$ in the function $k(r)$ in equation (C.11).

The dependence of $V_{\text {junction }}, V_{\text {total }} \equiv V_{D}+V_{\text {junction }}$ and $V_{\text {junction }} / V_{\text {total }}$ on the power $n_{k}$ is illustrated in figure $\mathrm{C} 4$. This figure shows that the contribution to the total volume from the junction region remains significantly nonzero when $n_{k}$ becomes large.

When $n_{k}$ goes to infinity and accordingly $\varepsilon$ goes to zero, one can obtain a lower bound of $V_{\text {junction }}$ as follows:

$$
\begin{gathered}
n_{k} \rightarrow \infty, \quad V_{\text {junction }} \rightarrow 4 \pi \int_{r_{k}-\varepsilon}^{r_{k}+\varepsilon} \frac{a^{2} r^{2}(a, r r) \mathrm{d} r}{\sqrt{1-k(r) r^{2}}} \\
>\frac{4 \pi a_{1}^{2} r_{k}^{3}}{\sqrt{1+r_{k}^{2}}} \int_{r_{k}-\varepsilon}^{r_{k}+\varepsilon} a, r \mathrm{~d} r
\end{gathered}
$$




$$
\begin{aligned}
& \cong \frac{4 \pi a_{1}^{2} r_{k}^{3}\left(a_{2}-a_{1}\right)}{\sqrt{1+r_{k}^{2}}} \\
& >0 \quad \text { if } a_{2}>a_{1},
\end{aligned}
$$

where $\varepsilon=\left(2 n_{k}\right)^{-1}$ and

$$
\begin{aligned}
& a_{1}(t) \equiv \lim _{n_{k} \rightarrow \infty} a\left(t, r_{k}-\varepsilon\right), \\
& a_{2}(t) \equiv \lim _{n_{k} \rightarrow \infty} a\left(t, r_{k}+\varepsilon\right) .
\end{aligned}
$$

(Note that the first line of equation (C.14) is obtained by keeping only the singular part in the integrand.) Thus, even when the junction region goes to a '2D surface' in the coordinate space, its volume remains significantly nonzero if the scale factor of the outer open under-density region $\left(a_{2}\right)$ is significantly larger than that of the inner closed over-density region $\left(a_{1}\right)$. As mentioned in the previous section, with the initial condition in equation (C.7) we do have $a_{2}>a_{1}$ for $t>0$.

Note that by changing the initial condition for $a_{1}(t)$ and $a_{2}(t)$ (instead of using that in equation (C.7)) Nambu and Tanimoto's treatment could become valid during some specific time period when $a_{1} \simeq a_{2}$, which might be achieved through, for example, choosing nontrivial $t_{b}(r)$ (i.e., making the big bang or the expansion at different places begin at different times). This may be the reason why non-trivial $t_{b}(r)$ is a necessary ingredient in our search for domain acceleration.

\section{References}

[1] Perlmutter S et al (Supernova Cosmology Project Collaboration) 1999 Astrophys. J. 517565 (Preprint astro-ph/9812133)

[2] Riess A G et al (Supernova Search Team Collaboration) 1998 Astron. J. 1161009 (Preprint astro-ph/9805201)

[3] Tonry J L et al (Supernova Search Team Collaboration) 2003 Astrophys. J. 5941 (Preprint astro-ph/0305008)

[4] Knop R A et al (The Supernova Cosmology Project Collaboration) 2003 Astrophys. J. 598102 (Preprint astro-ph/0309368)

[5] Barris B J et al 2004 Astrophys. J. 602571 (Preprint astro-ph/0310843)

[6] Riess A Get al (Supernova Search Team Collaboration) 2004 Astrophys. J. 607665 (Preprint astro-ph/0402512)

[7] Riess A G et al 2007 Astrophys. J. 65998 (Preprint astro-ph/0611572)

[8] Komatsu E et al (WMAP Collaboration) 2008 Preprint arXiv:0803.0547

[9] Ellis G F R 1984 General Relativity and Gravitation ed B Bertotti et al (Dordrecht: Reidel)

[10] Gu Je-An 2007 Mod. Phys. Lett. A 222013

[11] Buchert T 2000 Gen. Rel. Grav. 9306 (Preprint gr-qc/0001056)

[12] Schwarz D J 2002 Preprint astro-ph/0209584

[13] Bene G, Czinner V and Vasuth M 2006 Mod. Phys. Lett. A 211117 (Preprint astro-ph/0308161)

[14] Rasanen S 2004 J. Cosmol. Astropart. Phys. JCAP02(2004)003 (Preprint astro-ph/0311257)

[15] Rasanen S 2004 J. Cosmol. Astropart. Phys. JCAP11(2004)010 (Preprint gr-qc/0408097)

[16] Kolb E W, Matarrese S, Notari A and Riotto A 2005 Phys. Rev. D 71023524 (Preprint hep-ph/0409038)

[17] Barausse E, Matarrese S and Riotto A 2005 Phys. Rev. D 71063537 (Preprint astro-ph/0501152)

[18] Kolb E W, Matarrese S, Notari A and Riotto A 2008 Preprint hep-th/0503117

[19] Notari A 2006 Mod. Phys. Lett. A 212997 (Preprint astro-ph/0503715)

[20] Rasanen S 2006 Class. Quantum Grav. 231823 (Preprint astro-ph/0504005)

[21] Kolb E W, Matarrese S and Riotto A 2006 New J. Phys. 8322 (Preprint astro-ph/0506534)

[22] Mansouri R 2005 Preprint astro-ph/0512605

[23] Partovi M H and Mashhoon B 1984 Astrophys. J. 2764

[24] Mashhoon B 1984 The Big Bang and Georges Lemaitre ed A Barger (Dordrecht: Reidel)

[25] Mustapha N, Hellaby C and Ellis G F R 1997 Mon. Not. R. Astron. Soc. 292817 (Preprint gr-qc/9808079)

[26] Celerier M N 2000 Astron. Astrophys. 35363 (Preprint astro-ph/9907206) 
[27] Celerier M N 2002 Proc. XXXVth Rencontres de Moriond, Energy Densities in the Universe ed J Tran Thanh Van, R Ansari and Y Giraud-Heraud (Vietnam: Gioi) (Preprint astro-ph/0006273)

[28] Tomita K 2001 Mon. Not. R. Astron. Soc. 326287 (Preprint astro-ph/0011484)

[29] Iguchi H, Nakamura T and Nakao K i 2002 Prog. Theor. Phys. 108809 (Preprint astro-ph/0112419)

[30] Alnes H, Amarzguioui M and Gron O 2006 Phys. Rev. D 73083519 (Preprint astro-ph/0512006)

[31] Apostolopoulos P S, Brouzakis N, Tetradis N and Tzavara E 2006 J. Cosmol. Astropart. Phys. JCAP06(2006)009 (Preprint astro-ph/0603234)

[32] Garfinkle D 2006 Class. Quantum Grav. 234811 (Preprint gr-qc/0605088)

[33] Biswas T, Mansouri R and Notari A 2007 J. Cosmol. Astropart. Phys. JCAP12(2007)017 (Preprint astro-ph/0606703)

[34] Enqvist K and Mattsson T 2007 J. Cosmol. Astropart. Phys. JCAP02(2007)019 (Preprint astro-ph/0609120)

[35] Sicka C, Buchert T and Kerscher M 1999 Preprint astro-ph/9907137

[36] Buchert T, Kerscher M and Sicka C 2000 Phys. Rev. D 62043525 (Preprint astro-ph/9912347)

[37] Rasanen S 2008 J. Cosmol. Astropart. Phys. JCAP04(2008)026 (Preprint arXiv:0801.2692)

[38] Siegel E R and Fry J N 2005 Astrophys. J. 628 L1 (Preprint astro-ph/0504421)

[39] Ishibashi A and Wald R M 2006 Class. Quantum Grav. 23235 (Preprint gr-qc/0509108)

[40] Kasai M, Asada H and Futamase T 2006 Prog. Theor. Phys. 115827 (Preprint astro-ph/0602506)

[41] Vanderveld R A, Flanagan E E and Wasserman I 2007 Phys. Rev. D 76083504 (Preprint arXiv:0706.1931)

[42] Rasanen S 2006 J. Cosmol. Astropart. Phys. JCAP11(2006)003 (Preprint astro-ph/0607626)

[43] Kolb E W, Matarrese S and Riotto A 2005 Preprint astro-ph/0511073

[44] Lemaitre G 1933 Ann. Soc. Sci. Brux. Ser. I Sci. Math. Astron. Phys. A 5351

[45] Tolman R C 1934 Proc. Nat. Acad. Sci. 20169

[46] Bondi H 1947 Mon. Not. R. Astron. Soc. 107410

[47] Kai T, Kozaki H, Nakao K-i, Nambu Y and Yoo C M 2007 Prog. Theor. Phys. 117229 (Preprint gr-qc/0605120)

[48] Paranjape A and Singh T P 2006 Class. Quantum Grav. 236955 (Preprint astro-ph/0605195)

[49] Nambu Y and Tanimoto M 2005 Preprint gr-qc/0507057

[50] Rasanen S 2006 Int. J. Mod. Phys. D 152141 (Preprint astro-ph/0605632)

[51] Wiltshire D L 2007 Phys. Rev. Lett. 99251101 (Preprint arXiv:0709.0732)

[52] Paranjape A and Singh T P 2008 J. Cosmol. Astropart. Phys. JCAP03(2008)023 (Preprint arXiv:0801.1546)

[53] Buchert T 2000 Gen. Rel. Grav. 32105 (Preprint gr-qc/9906015)

[54] Palle D 2002 Nuovo Cimento B 117687 (Preprint astro-ph/0205462)

[55] Flanagan E E 2005 Phys. Rev. D 71103521 (Preprint hep-th/0503202)

[56] Hirata C M and Seljak U 2005 Phys. Rev. D 72083501 (Preprint astro-ph/0503582)

[57] Giovannini M 2006 Phys. Lett. B 6341 (Preprint hep-th/0505222)

[58] Alnes H, Amarzguioui M and Gron O 2007 J. Cosmol. Astropart. Phys. JCAP01(2007)007 (Preprint astro-ph/0506449)

[59] Hellaby C and Lake K 1985 Astrophys. J. 290381

[60] Li N and Schwarz D J 2007 Preprint arXiv:0710.5073 